\title{
Existence of periodic solutions to nonlinear $p$-regular boundary value problem
}

Beata Medak ${ }^{* *}$ and Alexey A Tret'yakov ${ }^{1,2,3}$

\section{"Correspondence:}

bmedak@uph.edu.pl

'Siedlce University of Natural

Sciences and Humanities, 3-go

Maja 54, Siedlce, 08-110, Poland

Full list of author information is

available at the end of the article

\section{照 Springer}

\begin{abstract}
The paper studies the existence problem of periodic solutions of the nonlinear dynamical systems in the singular case. We prove a certain generalization of the Andronov-Hopf theorem. This generalization is based on an application of the theorem on a modified $p$-factor operator. It also uses some other results and constructions of the $p$-regularity theory. Moreover, we prove theorems on the solution's uniqueness. We illustrate our results by the example of a nonlinear dynamical system of ordinary differential equations. Our purpose is to find periodic solutions of such system with fixed period $2 \pi$. This is a new research in relation to previous work, where the authors were looking for periodic solutions with period near $2 \pi$.
\end{abstract}

MSC: $37 \mathrm{C05} ; 37 \mathrm{C} 27 ; 37 \mathrm{G} 10$

Keywords: $p$-regularity; $p$-factor operator; contraction; dynamical systems; bifurcations

\section{Introduction}

In this paper we study the structure of periodic solutions of dynamical systems and bifurcation problems associated with such systems, i.e., we consider a nonlinear system of differential equations of the form

$$
\dot{u}=f(\mu, u), \quad u(0)=u(\tau),
$$

where $f \in \mathbb{C}^{3}\left(\mathbb{R} \times \mathbb{R}^{n}, \mathbb{R}^{n}\right), \tau, \mu \in \mathbb{R}, u \in \mathbb{C}^{1}\left(\mathbb{R}, \mathbb{R}^{n}\right)$, and $f(\mu, 0)=0$. Writing it as

$$
F(\mu, u)=\frac{d u}{d t}-f(\mu, u)=0,
$$

we apply to it the $p$-regularity theory [1-4].

Our applications refer to Andronov-Hopf bifurcations, which were considered by Buchner et al. [5], and by Tan [6]. In our paper the $p$-regularity theory will be applied to finding periodic solutions with the fixed period $\tau=2 \pi$. In the cited works the period is near $2 \pi$.

The issues related to the so-called Andronov-Hopf bifurcation involve the study of the bifurcation of periodic solutions of the dynamical systems (1), where $f(\mu, \bar{u})=0$ for some $\bar{u} \in \mathbb{R}^{n}$ and any $\mu \in \mathbb{R}$. By the changing of variables $\mu$ and $u$ in (1), the above problem

(c) 2015 Medak and Tret'yakov. This article is distributed under the terms of the Creative Commons Attribution 4.0 International License (http://creativecommons.org/licenses/by/4.0/), which permits unrestricted use, distribution, and reproduction in any medium, provided you give appropriate credit to the original author(s) and the source, provide a link to the Creative Commons license, and indicate if changes were made. 
reduces to a study of the solutions in a neighborhood of the point $(0,0)$. Then the system (1) has a family of equilibrium solutions $\{(\mu, 0): \mu \in \mathbb{R}\}$. Let $L_{0}:=\frac{\partial f}{\partial u}(0,0)$. The question is whether $(0,0)$ is a point of bifurcation. In this case, the sufficient conditions for bifurcation is the Andronov-Hopf result, which we can formulate as follows (see [5-8]): a bifurcation of periodic solutions of system (1) (i.e., $(0,0)$ is a bifurcation point) in a finite dimensional case occurs under the following assumptions:

1. $\pm i$ are algebraically simple eigenvalues of $L_{0}$ and $\pm k i$ do not belong to spectrum of $L_{0}$ for $k=0,2,3, \ldots$;

2. if $\alpha$ is an eigenvector of $L_{0}$ with eigenvalue $i$, then there exist $C^{2}$ functions $\beta(\mu)$ and $z(\mu)$ defined by $\frac{\partial f}{\partial u}(\mu, 0) z(\mu)=\beta(\mu) z(\mu), \beta(0)=i, z(0)=\alpha$ satisfying the Andronov-Hopf condition

$$
\operatorname{Re} \beta^{\prime}(0) \neq 0
$$

i.e., the eigenvalues cross the imaginary axis transversally.

It means that the conditions 1 and 2 are sufficient for $(0,0)$ to be a point of bifurcation. Our problem is to find periodic solutions of (1) with fixed period $\tau=2 \pi$. We do not use the classical Andronov-Hopf condition. Looking for methods of solving nonlinear equations we will show how we can modify the $p$-factor operator to provide sufficient conditions for the existence of nontrivial solutions. It turns out that the apparatus of $p$-regularity theory gives us the ability to construct a wide class of $p$-factor operators, by means of which one can describe the tangent cone to the sets of solutions and consequently get the solutions (see $[9,10])$. We will call such operators modified or generalized.

In this paper we prove a new theorem on the modified $p$-factor operator which is some generalization of the Andronov-Hopf theorem. In this generalization we do not require that the Andronov-Hopf condition $\operatorname{Re} \beta^{\prime}(0) \neq 0$ holds, i.e., we weaken this classical theorem. Essential is the fact of surjectivity of the $p$-factor operator. Note also that the method of proving will be based on the multimapping contraction principle. Moreover, the new theorems on the solution's uniqueness will be proved too. These results we can consider as a contribution to and a novelty in nonlinear differential equations theory that we represent in our paper.

\section{Structure of the paper}

The organization of the paper is as follows. We begin with some notations. Then we recall the main concept of $p$-regularity theory and some important lemmas which will be used later. In the next section we formulate and prove the theorems on solutions uniqueness. The main result of the paper is a theorem, which we called a generalization of AndronovHopf theorem. It is based on some modified (generalized) $p$-factor operator which allows us to introduce a new method of solving differential equations. We illustrate the theorem by an example including a nonlinear dynamical system of ordinary differential equations.

\section{Notations}

Suppose $X$ and $Y$ are Banach spaces and denote the space of all continuous linear operators from $X$ to $Y$ by $\mathcal{L}(X, Y)$. Let $p$ be a natural number and let $B: X \times X \times \cdots \times$ $X(p$ copies of $X) \rightarrow Y$ be a continuous symmetric $p$-multilinear mapping. The $p$-form associated to $B$ is the mapping $B[\cdot]^{p}: X \rightarrow Y$ defined by $B[x]^{p}=B(x, x, \ldots, x)$ for $x \in X$. 
Alternatively we may simply view $B[\cdot]^{p}$ as a homogeneous polynomial $Q: X \rightarrow Y$ of degree $p$, i.e., $Q(\alpha x)=\alpha^{p} Q(x)$. Throughout this paper, we assume that $F \in \mathcal{C}^{p}(X, Y)$, i.e., the mapping $F: X \rightarrow Y$ is $p$-times continuously Fréchet differentiable on $X$ and its $p$ th-order derivative at $x \in X$ will be denoted as $F^{(p)}(x)$ (a symmetric multilinear mapping of $p$ copies of $X$ to $Y$ ) and the associated $p$-form, also called a $p$ th-order mapping, is

$$
F^{(p)}(x)[h]^{p}=F^{(p)}(x)[h, h, \ldots, h] .
$$

In this paper we will write the vector $h$ as the row or column vector, depending on the situation.

We also use the notation

$$
\operatorname{Ker}^{p} F^{(p)}(x)=\left\{h \in X: F^{(p)}(x)[h]^{p}=0\right\}
$$

and refer to it as the $p$-kernel of the $p$ th-order mapping. Note that this set is a (non-convex) closed cone.

The set $M=M\left(x^{*}\right)=\left\{x \in X: F(x)=F\left(x^{*}\right)=0\right\}$ is called the solution set for the mapping $F$. We call $h$ a tangent vector to the set $M \subseteq X$ at $x^{*} \in M$ if there exist $\varepsilon>0$ and a function $r:[0, \varepsilon] \rightarrow X$ with the property that for $t \in[0, \varepsilon]$ we have $x^{*}+t h+r(t) \in M$ and $\|r(t)\|=o(t)$. The set of all tangent vectors at $x^{*}$ is called the tangent cone to $M$ at $x^{*}$ and is denoted by $T_{x^{*}} M$ (see [1]). A mapping $F: X \rightarrow Y$ is regular at $x^{*} \in X$ if $\operatorname{Im} F^{\prime}\left(x^{*}\right)=Y$. In the regular case, the tangent cone to the solution set coincides with the kernel of the first derivative of the mapping $F$. Recall the following theorem.

Theorem 1 (Classical Lyusternik theorem [1]) Let $X$ and $Y$ be the Banach spaces and let the mapping $F: X \rightarrow Y$ be regular at $x^{*} \in X$. Then

$$
T_{x^{*}} M=\operatorname{Ker} F^{\prime}\left(x^{*}\right)
$$

The notion of regularity is generalized to the notion of the so-called $p$-regularity.

\section{Elements of $p$-regularity theory}

Consider the nonlinear problem

$$
F(x)=0,
$$

where $F$ is a sufficiently smooth mapping between Banach spaces $X$ and $Y$. If a solution $x^{*}$ of this equation is regular, i.e., the operator $F^{\prime}\left(x^{*}\right)$ is surjective, then the above equation describes a regular submanifold of $X$ near the point $x^{*}$.

The $p$-regularity theory $[1-4,11-14]$ deals with irregular cases. In [4], it was shown that the notions of nonlinearity and irregularity are strongly connected. The main idea of our $p$-regularity construction is to replace the operator $F^{\prime}\left(x^{*}\right)$ (which is not surjective) with another linear operator (constructed by means of the first- and higher-order derivatives) which is surjective. The latter operator is denoted by $\Psi_{p}\left(x^{*}, h\right)$. Here the vector $h$ belongs to the tangent cone to the set $\{x \in X: F(x)=0\}$ at $x^{*}$ and $p$ is taken so large (if it ever exists) that the operator $\Psi_{p}\left(x^{*}, h\right)$ turns out to be surjective (the so-called $p$-regularity condition). Assume that $x^{*} \in U \subseteq X, U$ is a neighborhood of the element $x^{*}$. Let a mapping $F: U \rightarrow$ $Y$ be $p$-times Frechet differentiable in $U(p>1)$ and $\operatorname{Im} F^{\prime}\left(x^{*}\right) \neq Y$ (the regularity condition 
does not hold). To define the notion of $p$-regularity, let us first define the so-called $p$-factor operator (see [1]). Assume that the space $Y$ is decomposed into a direct sum

$$
Y=Y_{1} \oplus \cdots \oplus Y_{p}
$$

where $Y_{1}=\operatorname{cl}\left(\operatorname{Im} F^{\prime}\left(x^{*}\right)\right)$ (the closure of the image of the first derivative of $F$ evaluated at $x^{*}$ ) and the next spaces are defined as follows. Let $Z_{2}$ be a closed complementary subspace to $Y_{1}$, that is, $Y=Y_{1} \oplus Z_{2}$ (we assume that such a closed complement exists), and let $P_{Z_{2}}: Y \rightarrow$ $Z_{2}$ be the projection operator onto $Z_{2}$ along $Y_{1}$. Let $Y_{2}=\operatorname{cl}\left(\operatorname{span} \operatorname{Im} P_{Z_{2}} F^{\prime \prime}\left(x^{*}\right)[\cdot]^{2}\right) \subseteq Z_{2}$ (the closed linear span of the image of the quadratic mapping $\left.P_{Z_{2}} F^{\prime \prime}\left(x^{*}\right)[\cdot]^{2}\right)$. More generally, define

$$
Y_{i}=\operatorname{cl}\left(\operatorname{span} \operatorname{Im} P_{Z_{i}} F^{(i)}\left(x^{*}\right)[\cdot]^{i}\right) \subseteq Z_{i}, \quad i=2, \ldots, p-1,
$$

where $Z_{i}$ is a closed complementary subspace to $Y_{1} \oplus \cdots \oplus Y_{i-1}, i=2, \ldots, p$ with respect to $Y$, and $P_{Z_{i}}: Y \rightarrow Z_{i}$ is a projection operator onto $Z_{i}$ along $Y_{1} \oplus \cdots \oplus Y_{i-1}, i=2, \ldots, p$ with respect to $Y$. Finally, let $Y_{p}=Z_{p}$. The order $p$ is chosen as the minimal number (if it exists) for which the above decomposition (4) holds.

Now, let us define the following mappings:

$$
f_{i}: U \rightarrow Y_{i}, \quad f_{i}(x)=\Pi_{i} F(x), \quad i=1, \ldots, p
$$

where $\Pi_{i}: Y \rightarrow Y_{i}$ is a projection operator along $Y_{1} \oplus \cdots \oplus Y_{i-1} \oplus Y_{i+1} \oplus \cdots \oplus Y_{p}$. Then the mapping $F$ can be represented as

$$
F(x)=f_{1}(x)+\cdots+f_{p}(x) .
$$

Sometimes it is more convenient to represent $Y$ as a product $Y_{1} \times \cdots \times Y_{p}$ instead of the sum $Y_{1} \oplus \cdots \oplus Y_{p}$. Then

$$
F(x)=\left(f_{1}(x), \ldots, f_{p}(x)\right)
$$

Let us recall some important definitions of $p$-regularity theory for the further considerations.

Definition 2 The linear operator mapping $X$ to $Y$,

$$
\Psi_{p}(h)=\Psi_{p}\left(x^{*}, h\right)=f_{1}^{\prime}\left(x^{*}\right)+f_{2}^{\prime \prime}\left(x^{*}\right)[h]+\cdots+f_{p}^{(p)}\left(x^{*}\right)[h]^{p-1}
$$

such that

$$
\Psi_{p}(h) x=\Psi_{p}\left(x^{*}, h\right) x=f_{1}^{\prime}\left(x^{*}\right) x+f_{2}^{\prime \prime}\left(x^{*}\right)[h] x+\cdots+f_{p}^{(p)}\left(x^{*}\right)[h]^{p-1} x, \quad x \in X,
$$

is called $p$-factor operator.

Here, by definition, $f_{2}^{\prime \prime}\left(x^{*}\right)[h] x=f_{2}^{\prime \prime}\left(x^{*}\right)[h, x]$, etc. 
Sometimes it is convenient to use the following equivalent definition of $p$-factor operator:

$$
\begin{aligned}
\Psi_{p}(h) & =\Psi_{p}\left(x^{*}, h\right)=\left(f_{1}^{\prime}\left(x^{*}\right), f_{2}^{\prime \prime}\left(x^{*}\right)[h], \ldots, f_{p}^{(p)}\left(x^{*}\right)[h]^{p-1}\right) \\
& =\left(\Pi_{1} F^{\prime}\left(x^{*}\right), \Pi_{2} F^{\prime \prime}\left(x^{*}\right)[h], \ldots, \Pi_{p} F^{(p)}\left(x^{*}\right)[h]^{p-1}\right)
\end{aligned}
$$

for $h \in X$.

We say that $F$ is completely degenerate at $x^{*}$ up to the order $p$ if $F^{(i)}\left(x^{*}\right)=0, i=1, \ldots, p-1$.

Remark 3 In the completely degenerate case the $p$-factor operator reduces to $F^{(p)}\left(x^{*}\right)[h]^{p-1}$.

Remark 4 For each mapping $f_{i}, i=1, \ldots, p$ we have ([1], p.145):

$$
f_{i}^{(k)}\left(x^{*}\right)=0, \quad k=1, \ldots, i-1, \forall i=1, \ldots, p
$$

Remark 5 According to the Remark 4 the expressions

$$
f_{i}^{(i)}\left(x^{*}\right)[h]^{i-1}=\Pi_{i} F^{(i)}\left(x^{*}\right)[h]^{i-1}, \quad i=1, \ldots, p,
$$

are $i$-factor operators corresponding to completely degenerate mappings $f_{i}$. So the general degeneration of $F$ can be reduced to the study of completely degenerated mappings $f_{i}$.

Sometimes we will be write round brackets $(\cdot)$ instead of square ones $[\cdot]$.

Definition 6 The $p$-kernel of the operator $\Psi_{p}(h)$ is a set

$$
\begin{aligned}
H_{p}\left(x^{*}\right) & =\operatorname{Ker}^{p} \Psi_{p}(h)=\left\{h \in X: \Psi_{p}(h)[h]=0\right\} \\
& =\left\{h \in X: f_{1}^{\prime}\left(x^{*}\right)[h]+f_{2}^{\prime \prime}\left(x^{*}\right)[h]^{2}+\cdots+f_{p}^{(p)}\left(x^{*}\right)[h]^{p}=0\right\} .
\end{aligned}
$$

Note that the following relation holds:

$$
\operatorname{Ker}^{p} \Psi_{p}(h)=\left\{\bigcap_{i=1}^{p} \operatorname{Ker}^{i} f_{i}^{(i)}\left(x^{*}\right)\right\} .
$$

Again, this set is a closed cone, in general non-convex.

Furthermore $p$-kernel of the operator $F^{(p)}\left(x^{*}\right)$ in the completely degenerate case is a set $\operatorname{Ker}^{p} F^{(p)}\left(x^{*}\right)=\left\{h \in X: F^{(p)}\left(x^{*}\right)[h]^{p}=0\right\}$.

Definition 7 A mapping $F$ is called $p$-regular at $x^{*}$ along $h(p>1)$ if $\operatorname{Im} \Psi_{p}(h)=Y$ (i.e., the operator $\Psi_{p}(h)$ is surjective).

Definition 8 A mapping $F$ is called $p$-regular at $x^{*}(p>1)$ if either it is $p$-regular along every $h \in H_{p}\left(x^{*}\right) \backslash\{0\}$ or $H_{p}\left(x^{*}\right)=\{0\}$.

The following theorem gives the description of the tangent cone to the solution set $M$ in the degenerate case. 
Theorem 9 (Generalized Lyusternik theorem [1]) Let $X$ and $Y$ be the Banach spaces and let the mapping $F \in C^{p}(X, Y)$ be p-regular at $x^{*} \in M$. Then

$$
T_{x^{*}} M=H_{p}\left(x^{*}\right)
$$

where $T_{x^{*}} M$ denotes the tangent cone to the set $M$ at the point $x^{*}$.

The above apparatus of $p$-regularity is a new tool for studying nonlinear problems.

\section{Some important lemmas}

In this section we present some lemmas which play important roles in the further considerations and will be used later.

Lemma 10 ([10]) Let $A_{1}, A_{2}, \ldots, A_{p} \in \mathcal{L}(X, Y), Y=Y_{1} \oplus \cdots \oplus Y_{p}$. Let $\operatorname{Im} \Pi_{k} A_{k}=Y_{k}$, where $\Pi_{k}: Y \rightarrow Y_{k}$ is a projection operator from the space $Y$ onto $Y_{k}$ along $Y_{1} \oplus \cdots \oplus Y_{k-1} \oplus$ $Y_{k+1} \oplus \cdots \oplus Y_{p}, k=1, \ldots, p$, and $\Pi_{1} A_{1}=A_{1}$. Then

$$
\left(\Pi_{1} A_{1}+\Pi_{2} A_{2}+\cdots+\Pi_{p} A_{p}\right) X=Y \quad \Leftrightarrow \quad\left(\Pi_{p} A_{p}\right)\left(\bigcap_{i=1}^{p-1} \operatorname{Ker} \Pi_{i} A_{i}\right)=Y_{p} .
$$

Let $X$ and $Y$ be Banach spaces. By the mapping $\Phi: X \rightarrow 2^{Y}$ we mean a multivalued mapping (multimapping) from $X$ to the set of all subsets of a space $Y$. Let $\rho(x, y)=\|x-y\|$ be the distance between elements $x$ and $y$ in a Banach space and let $\rho(x, M)=\inf \{\|x-z\|$ : $z \in M\}$ be the distance from element $x$ to subset $M$ in this space. $\operatorname{By}_{\operatorname{dist}_{H}}\left(M_{1}, M_{2}\right)=$ $\max \left\{\sup \left\{\rho\left(x, M_{2}\right): x \in M_{1}\right\}, \sup \left\{\rho\left(x, M_{1}\right): x \in M_{2}\right\}\right\}$ we denote the Hausdorff distance between the sets $M_{1}$ and $M_{2}$.

Lemma 11 (Multimapping contraction principle [15]) Let $Z$ be a Banach space. Assume that a multimapping

$$
\Phi: U_{\varepsilon}\left(z_{0}\right) \rightarrow 2^{Z}
$$

is given on a ball $U_{\varepsilon}\left(z_{0}\right)=\left\{z \in Z: \rho\left(z, z_{0}\right)<\varepsilon\right\}(\varepsilon>0)$, where the sets $\Phi(z)$ are non-empty and closed for any $z \in U_{\varepsilon}\left(z_{0}\right)$. Further, assume that there exists a number $\theta, 0<\theta<1$, such that

1. $\operatorname{dist}_{H}\left(\Phi\left(z_{1}\right), \Phi\left(z_{2}\right)\right) \leq \theta \rho\left(z_{1}, z_{2}\right)$ for any $z_{1}, z_{2} \in U_{\varepsilon}\left(z_{0}\right)$,

2. $\rho\left(z_{0}, \Phi\left(z_{0}\right)\right)<(1-\theta) \varepsilon$.

Then, for every number $\varepsilon_{1}$ satisfying the inequality

$$
\rho\left(z_{0}, \Phi\left(z_{0}\right)\right)<\varepsilon_{1}<(1-\theta) \varepsilon
$$

there exists $z \in B_{\varepsilon_{1} /(1-\theta)}\left(z_{0}\right):=\left\{\omega: \rho\left(\omega, z_{0}\right) \leq \varepsilon_{1} /(1-\theta)\right\}$ such that

$$
z \in \Phi(z)
$$

Moreover, among the points satisfying (7), there exists a point $z$ such that

$$
\rho\left(z, z_{0}\right) \leq \frac{2}{1-\theta} \rho\left(z_{0}, \Phi\left(z_{0}\right)\right) .
$$


For a linear surjective operator $\Lambda: X \rightarrow Y$ we denote by $\Lambda^{-1}$ its right inverse, that is, $\Lambda^{-1}: Y \rightarrow 2^{X}$, which takes an element $y \in Y$ to its complete inverse image of the mapping $\Lambda, \Lambda^{-1} y=\{x \in X: \Lambda x=y\}$, and of course $\Lambda \Lambda^{-1}=I_{Y}$. We set $\left\|\Lambda^{-1} y\right\|=\inf \left\{\|x\|: x \in \Lambda^{-1} y\right\}$. By the 'norm' of such a right inverse operator we mean the number

$$
\left\|\Lambda^{-1}\right\|=\sup _{\|y\|=1}\left\|\Lambda^{-1} y\right\|
$$

By the Banach theorem on surjective linear operator, we have $\left\|\Lambda^{-1}\right\|<\infty$. Note that if $\Lambda$ is one-to-one, then $\left\|\Lambda^{-1}\right\|$ is the usual norm of the operator $\Lambda^{-1}$. In our considerations, by $\Lambda^{-1}$ we shall mean just the right inverse multivalued operator with the norm defined by $(8)$.

Lemma 12 ([16]) Let $X$ and $Y$ be the Banach spaces, $F: X \rightarrow Y$ nonlinear operator, $F \in$ $C^{p}(X, Y)$. Let $Y=Y_{1} \oplus \cdots \oplus Y_{p}$, where $Y_{i}, i=1, \ldots, p$ are the Banach spaces. Let $y \in Y$, $y=y_{1}+\cdots+y_{p}$, where $y_{i} \in Y_{i}, i=1, \ldots, p$, and let $h \in X,\|h\|=1$. Assume that

$$
\left\|\left\{F^{\prime}\left(x^{*}\right)+\Pi_{2} F^{\prime \prime}\left(x^{*}\right)[h]+\cdots+\Pi_{p} F^{(p)}\left(x^{*}\right)[h]^{p-1}\right\}^{-1}\right\|:=c<\infty
$$

where $\Pi_{k}$ are as in Lemma 10. Then for all nonzero $\alpha_{i} \in \mathbb{R}, i=1, \ldots, p$, and all nonzero $t \in \mathbb{R}$ we have

$$
\begin{aligned}
& \left\|\left\{\alpha_{1} F^{\prime}\left(x^{*}\right)+\alpha_{2} \Pi_{2} F^{\prime \prime}\left(x^{*}\right)[t h]+\cdots+\alpha_{p} \Pi_{p} F^{(p)}\left(x^{*}\right)[t h]^{p-1}\right\}^{-1}\left(y_{1}+\cdots+y_{p}\right)\right\| \\
& \quad \leq c\left(\frac{1}{\alpha_{1}}\left\|y_{1}\right\|+\frac{1}{\alpha_{2} t}\left\|y_{2}\right\|+\cdots+\frac{1}{\alpha_{p} t^{p-1}}\left\|y_{p}\right\|\right) .
\end{aligned}
$$

Lemma 13 (Mean value theorem [15]) Let X, $Y$ be Banach spaces, $U$ an open subset of the space $X,[x, x+\Delta]$ a closed segment in $U$. If $F: U \rightarrow Y$ and $F \in \mathcal{C}^{1}([x, x+\Delta])$, then for any $\Lambda \in \mathcal{L}(X, Y)$ we have

$$
\|F(x+\Delta)-F(x)-\Lambda \Delta\| \leq \sup _{\theta \in[0,1]}\left\|F^{\prime}(x+\theta \Delta)-\Lambda\right\| \cdot\|\Delta\|
$$

Lemma 14 Let $X, Y$ be the vector spaces, $B[\cdot]^{p}: X \rightarrow Y$ be the homogeneous $p$-form defined on the space $X$ associated to a continuous, symmetric, $p$-multilinear mapping $B: X \times \cdots \times$ $X(p$ copies of $X) \rightarrow Y$. Then

$$
\begin{aligned}
& B^{(p)}[h]^{p}=p ! B[h]^{p}, \\
& B^{(p)}[h]^{p-1}=(p-1) !\left(B[h]^{p}\right)^{\prime},
\end{aligned}
$$

where $h \in X$ and $B^{(p)}$ denotes the pth-order derivative of the mapping $B[\cdot]^{p}$.

The proof of this lemma follows from the properties of a homogeneous $p$-form (see $[2,17])$.

Lemma 15 Let for any $s \in(0, \delta)$, where $\delta>0$ is sufficiently small, the linear operator $A+s B$ be a surjection, then the linear operator $A+P_{\operatorname{Im} A^{\perp}} B$ is a surjection.

The proof of the above lemma is in [9]. 


\section{Theorems on solution's uniqueness}

The generalized Lyusternik theorem ensures the existence of a tangent line from the $p$-kernel of the $p$-factor operator $\Psi_{p}\left(x^{*}, h\right)$, which determines a solution of the equation $F(x)=0$. In the completely degenerate case, where $F^{(k)}\left(x^{*}\right)=0$ for $k=1, \ldots, p-1$, we understand it in the following sense. If the element $h \in \operatorname{Ker}^{p} F^{(p)}\left(x^{*}\right)$, then there exists $r(t)$ such that $\|r(t)\|=o(t)$ and $x^{*}+t h+r(t) \in M$, i.e., $F\left(x^{*}+t h+r(t)\right)=0$. However, the guarantee of the existence of a solution does not mean that the solution is unique. Below we show that under additional conditions we can consider the problem of the solution's uniqueness. So, let us take into account the completely degenerate case and begin with the following important remark. In this section will be considered only the finite dimensional case, i.e., $X=\mathbb{R}^{n}, Y=\mathbb{R}^{n-1}$, and $F \in C^{p+1}\left(\mathbb{R}^{n}, \mathbb{R}^{n-1}\right)$.

Remark 16 Let $h \in \operatorname{Ker}^{p} F^{(p)}\left(x^{*}\right)$. If the curve $x(t)=x^{*}+t h+r(t)$ is a solution of the equation $F(x)=0$, i.e., $F\left(x^{*}+t h+r(t)\right)=0$, and $\|r(t)\|=o(t)$ for $t \in(-\varepsilon, \varepsilon), \varepsilon>0$, then $r(t)$ always can be regarded as an element belonging to the orthogonal subspace to the space $\{t h: t \in \mathbb{R}\}$.

Now we introduce the definition.

Definition 17 We say that a solution (or curve) $x(t)=x^{*}+t h+r(t)$ of equation $F(x)=0$ is unique for some vector $h \in \operatorname{Ker}^{p} F^{(p)}\left(x^{*}\right)$, if there exists exactly one curve $x(t)=x^{*}+t h+r(t)$ (exactly unique function $r(t)$ ), such that $F\left(x^{*}+t h+r(t)\right)=0$ and $r(t) \perp h$, where $\|r(t)\|=o(t)$ for $t \in(-\varepsilon, \varepsilon)$ and $\varepsilon>0$ is sufficiently small.

We shall prove the following lemma.

Lemma 18 Let $F \in C^{p+1}\left(\mathbb{R}^{n}, \mathbb{R}^{n-1}\right)$ and $F\left(x^{*}\right)=0, F^{(k)}\left(x^{*}\right)=0$ for $k=1, \ldots, p-1$, $F^{(p)}\left(x^{*}\right)[h]^{p-1}\left(\mathbb{R}^{n}\right)=\mathbb{R}^{n-1}$, where $h \in \operatorname{Ker}^{p} F^{(p)}\left(x^{*}\right)$. Then there exists a unique curve $x^{*}+$ th $+r(t), r(t) \perp h, t \in(-\varepsilon, \varepsilon), \varepsilon>0$ such that

$$
F\left(x^{*}+t h+r(t)\right)=0, \quad\|r(t)\|=o(t) .
$$

Proof Assume that $h \in \operatorname{Ker}^{p} F^{(p)}\left(x^{*}\right)$. Moreover, suppose that there exist a sequence $\left\{t_{k}\right\}_{k \in \mathbb{N}}, t_{k} \rightarrow 0$ and two solutions $x^{*}+t_{k} h+r_{1}\left(t_{k}\right), x^{*}+t_{k} h+r_{2}\left(t_{k}\right)$ such that $F\left(x^{*}+\right.$ $\left.t_{k} h+r_{1}\left(t_{k}\right)\right)=F\left(x^{*}+t_{k} h+r_{2}\left(t_{k}\right)\right)=0$, where $r_{1}\left(t_{k}\right) \neq r_{2}\left(t_{k}\right), r_{1}(t), r_{2}(t) \perp h,\left\|r_{1}\left(t_{k}\right)\right\|=o\left(t_{k}\right)$ and $\left\|r_{2}\left(t_{k}\right)\right\|=o\left(t_{k}\right), k=1,2, \ldots$ Next, for the sake of simplicity, let us denote $r_{1}:=r_{1}(t)$, $r_{2}:=r_{2}(t)$, where $t:=t_{k}$.

We apply an expansion of the $F\left(x^{*}+t h+r_{2}\right)$ by the Taylor formula. We have

$$
\begin{aligned}
0= & F\left(x^{*}+t h+r_{2}\right) \\
= & F\left(x^{*}+t h+r_{1}\right)+F^{\prime}\left(x^{*}+t h+r_{1}\right)\left(r_{2}-r_{1}\right) \\
& +\frac{1}{2} F^{\prime \prime}\left(x^{*}+t h+r_{1}\right)\left(r_{2}-r_{1}\right)^{2}+\cdots+\frac{1}{p !} F^{(p)}\left(x^{*}+t h+r_{1}\right)\left(r_{2}-r_{1}\right)^{p} \\
& +\xi\left(r_{2}-r_{1}\right),
\end{aligned}
$$

where $\xi: \mathbb{R}^{n} \rightarrow \mathbb{R}^{n-1}$ and $\left\|\xi\left(r_{2}-r_{1}\right)\right\|=O_{\mathbb{R}^{n-1}}\left(\left\|r_{2}-r_{1}\right\|^{p+1}\right)\left(O_{\mathbb{R}^{n-1}}(t)\right.$ denotes $O$ large in the space $\mathbb{R}^{n-1}$ ). 
Moreover,

$$
\begin{aligned}
F^{\prime}\left(x^{*}+t h+r_{1}\right)= & F^{\prime}\left(x^{*}\right)+F^{\prime \prime}\left(x^{*}\right)\left(t h+r_{1}\right)+\frac{1}{2} F^{\prime \prime \prime}\left(x^{*}\right)\left(t h+r_{1}\right)^{2} \\
& +\cdots+\frac{1}{(p-1) !} F^{(p)}\left(x^{*}\right)\left(t h+r_{1}\right)^{p-1}+O_{\mathbb{R}^{n-1}}\left(t^{p}\right)
\end{aligned}
$$

and

$$
\begin{aligned}
F^{\prime \prime}\left(x^{*}+t h+r_{1}\right)= & F^{\prime \prime}\left(x^{*}\right)+F^{\prime \prime \prime}\left(x^{*}\right)\left(t h+r_{1}\right)+\frac{1}{2} F^{(4)}\left(x^{*}\right)\left(t h+r_{1}\right)^{2} \\
& +\cdots+\frac{1}{(p-2) !} F^{(p)}\left(x^{*}\right)\left(t h+r_{1}\right)^{p-2}+O_{\mathbb{R}^{n-1}}\left(t^{p-1}\right),
\end{aligned}
$$

and continuing

$$
F^{(p)}\left(x^{*}+t h+r_{1}\right)=F^{(p)}\left(x^{*}\right)+O_{\mathbb{R}^{n-1}}(t) .
$$

Substituting (15), (16), and (17) into (14) and applying the conditions $F^{(k)}\left(x^{*}\right)=0$ for $k=1, \ldots, p-1$, we have

$$
\begin{aligned}
& \left(\frac{1}{(p-1) !} F^{(p)}\left(x^{*}\right)\left(t h+r_{1}\right)^{p-1}+O_{\mathbb{R}^{n-1}}\left(t^{p}\right)\right)\left(r_{2}-r_{1}\right) \\
& \quad+\left(\frac{1}{2(p-2) !} F^{(p)}\left(x^{*}\right)\left(t h+r_{1}\right)^{p-2}+O_{\mathbb{R}^{n-1}}\left(t^{p-1}\right)\right)\left(r_{2}-r_{1}\right)^{2}+\cdots \\
& \quad+\left(\frac{1}{p !} F^{(p)}\left(x^{*}\right)+O_{\mathbb{R}^{n-1}}(t)\right)\left(r_{2}-r_{1}\right)^{p}+\xi\left(r_{2}-r_{1}\right)=0 .
\end{aligned}
$$

Dividing two sides of this equation by $t^{p-1}\left\|r_{2}-r_{1}\right\|$, and introducing the notation $A$, we obtain

$$
\begin{aligned}
A:= & \left(\frac{1}{(p-1) !} F^{(p)}\left(x^{*}\right)\left(t h+r_{1}\right)^{p-1}+O_{\mathbb{R}^{n-1}}\left(t^{p}\right)\right) \frac{\left(r_{2}-r_{1}\right)}{t^{p-1}\left\|r_{2}-r_{1}\right\|} \\
& +\left(\frac{1}{2(p-2) !} F^{(p)}\left(x^{*}\right)\left(t h+r_{1}\right)^{p-2}+O_{\mathbb{R}^{n-1}}\left(t^{p-1}\right)\right) \frac{\left(r_{2}-r_{1}\right)^{2}}{t^{p-1}\left\|r_{2}-r_{1}\right\|}+\cdots \\
& +\left(\frac{1}{p !} F^{(p)}\left(x^{*}\right)+O_{\mathbb{R}^{n-1}}(t)\right) \frac{\left(r_{2}-r_{1}\right)^{p}}{t^{p-1}\left\|r_{2}-r_{1}\right\|}+\frac{\xi\left(r_{2}-r_{1}\right)}{t^{p-1}\left\|r_{2}-r_{1}\right\|}=0 .
\end{aligned}
$$

We can represent the above equation as

$$
\frac{1}{(p-1) !} F^{(p)}\left(x^{*}\right)\left(t h+r_{1}\right)^{p-1} \frac{\left(r_{2}-r_{1}\right)}{t^{p-1}\left\|r_{2}-r_{1}\right\|}+\omega(t)=0,
$$

where $\omega: \mathbb{R} \rightarrow \mathbb{R}^{n-1}, \omega(t) \rightarrow 0$ as $t \rightarrow 0$.

This yields

$$
\frac{1}{(p-1) !} F^{(p)}\left(x^{*}\right)(t h)^{p-1} \frac{\left(r_{2}-r_{1}\right)}{t^{p-1}\left\|r_{2}-r_{1}\right\|}+\sigma(t)+\omega(t)=0,
$$

where $\sigma: \mathbb{R} \rightarrow \mathbb{R}^{n-1}, \sigma(t) \rightarrow 0$ as $t \rightarrow 0$.

We need to consider two cases. 
1. Assume that $F^{(p)}\left(x^{*}\right)(h)^{p-1} \frac{\left(r_{2}-r_{1}\right)}{\left\|r_{2}-r_{1}\right\|} \rightarrow 0$ as $t \rightarrow 0$, where $r_{1}=r_{1}(t), r_{2}=r_{2}(t)$.

This implies that there exists an element $\bar{\eta}$, such that $\|\bar{\eta}\|=1$ and

$$
F^{(p)}\left(x^{*}\right)(h)^{p-1} \bar{\eta}=0
$$

Moreover, $\bar{\eta} \perp \operatorname{span}\{h\}$.

This is contrary to the fact that the operator $F^{(p)}\left(x^{*}\right)(h)^{p-1}$ is a surjection, since the kernel of $F^{(p)}\left(x^{*}\right)(h)^{p-1}$ is one-dimensional. More precisely, we obtained two elements $h$ and $\bar{\eta}$, for which $F^{(p)}\left(x^{*}\right)(h)^{p-1} h=0$ and $F^{(p)}\left(x^{*}\right)(h)^{p-1} \bar{\eta}=0$, then the kernel is two-dimensional, which contradicts the surjectivity of $F^{(p)}\left(x^{*}\right)(h)^{p-1}$.

2. Assume now that $F^{(p)}\left(x^{*}\right)(h)^{p-1} \frac{\left(r_{2}-r_{1}\right)}{\left\|r_{2}-r_{1}\right\|} \nrightarrow 0$ as $t \rightarrow 0$.

Therefore there is a subsequence $\left\{t_{k_{i}}\right\}_{i \in \mathbb{N}}$ of the sequence $\left\{t_{k}\right\}_{k \in \mathbb{N}}$, such that $\left\|F^{(p)}\left(x^{*}\right)(h)^{p-1} \frac{\left(r_{2}\left(t_{k_{i}}\right)-r_{1}\left(t_{k_{i}}\right)\right)}{\left\|r_{2}\left(t_{k_{i}}\right)-r_{1}\left(t_{k_{i}}\right)\right\|}\right\| \geq M>0$ as $t_{k_{i}} \rightarrow 0$.

Note that the conditions $\sigma\left(t_{k_{i}}\right) \rightarrow 0$ and $\omega\left(t_{k_{i}}\right) \rightarrow 0$ give

$$
\begin{aligned}
0 & =\|A\|=\left\|F^{(p)}\left(x^{*}\right)(h)^{p-1} \frac{\left(r_{2}\left(t_{k_{i}}\right)-r_{1}\right)\left(t_{k_{i}}\right)}{\left\|r_{2}\left(t_{k_{i}}\right)-r_{1}\left(t_{k_{i}}\right)\right\|}+\sigma\left(t_{k_{i}}\right)+\omega\left(t_{k_{i}}\right)\right\| \\
& \geq\left\|F^{(p)}\left(x^{*}\right)(h)^{p-1} \frac{\left(r_{2}\left(t_{k_{i}}\right)-r_{1}\left(t_{k_{k}}\right)\right)}{\left\|r_{2}\left(t_{k_{i}}\right)-r_{1}\left(t_{k_{i}}\right)\right\|}\right\|-\left\|\sigma\left(t_{k_{i}}\right)\right\|-\left\|\omega\left(t_{k_{i}}\right)\right\| \geq \frac{M}{2} \neq 0,
\end{aligned}
$$

which is impossible and the lemma is proved.

The above lemma can be generalized as follows.

Lemma 19 Let $F \in C^{p+1}\left(\mathbb{R}^{n}, \mathbb{R}^{n-1}\right), F\left(x^{*}\right)=0$, and $\Psi_{p}\left(x^{*}, h\right)\left(\mathbb{R}^{n}\right)=\mathbb{R}^{n-1}$, where $\Psi_{p}$ is $p$-factor operator and $h \in \operatorname{Ker}^{p} \Psi_{p}\left(x^{*}\right)$. Then there exists a unique curve $x^{*}+t h+r(t)$ such that

$$
F\left(x^{*}+t h+r(t)\right)=0
$$

and $\|r(t)\|=o(t), r(t) \perp h$ for all $t \in(-\varepsilon, \varepsilon)$, where $\varepsilon>0$ is sufficiently small.

Proof Note that according to the definition of the $p$-factor operator, i.e.,

$$
\Psi_{p}\left(x^{*}, h\right)=\left(\Pi_{1} F^{\prime}\left(x^{*}\right), \Pi_{2} F^{\prime \prime}\left(x^{*}\right)[h], \ldots, \Pi_{p} F^{(p)}\left(x^{*}\right)[h]^{p-1}\right),
$$

and Remark 5, the assumptions of Lemma 18 hold for the mapping of the form $\Pi_{j} F^{(j)}\left(x^{*}\right)[h]^{j-1}: \mathbb{R}^{n} \stackrel{\text { on }}{\rightarrow} \mathbb{R}^{n-1}, j=1, \ldots, p$. Each coordinate $\Pi_{k} F(x)$ is completely degenerate at the point $x^{*}$ and the $p$-factor operator is a surjection. Then analogously to the completely degenerate case one can prove that there exists a unique element $r(t) \perp h$. Therefore Lemma 19 is true.

Remark 20 The curve $x^{*}+t h+r(t)$ determined by the element $h$ from the $p$-kernel of the $p$-factor operator $\Psi_{p}$ is a unique solution of the equation $F(x)=0$, i.e., for a given $h$, such a curve is unique.

\section{Generalization of Andronov-Hopf theorem}

We will prove Theorem 21 on a generalized (modified) $p$-factor operator, which is certain generalization of Andronov-Hopf theorem [5]. 
Theorem 21 Let $X$ and $Y$ be Banach spaces, $F \in \mathcal{C}^{3}(X, Y)$. Assume that there exists $x^{*} \in X$, such that $F\left(x^{*}\right)=0, F^{\prime}\left(x^{*}\right)=0$ and there exist vectors $h$ and $\bar{h}$ in $X$, such that $Y_{1}:=\operatorname{Im} F^{\prime}\left(x^{*}\right)$ is closed and has a closed direct complement $Y_{1}^{\perp}, Y_{2}:=\operatorname{Im} \Pi_{Y_{1}^{\perp}} F^{\prime \prime}\left(x^{*}\right)[h]$ is closed and has a closed direct complement $Y_{2}^{\perp}$, and

$$
\begin{aligned}
& Y_{2}^{\perp}=\operatorname{Im} P_{\left(Y_{1} \oplus Y_{2}\right)^{\perp}}\left(F^{\prime \prime}\left(x^{*}\right)[\bar{h}]+\frac{1}{2} F^{\prime \prime \prime}\left(x^{*}\right)[h]^{2}\right), \\
& Y=Y_{1} \oplus Y_{2} \oplus Y_{2}^{\perp} .
\end{aligned}
$$

Moreover, assume that

$$
\begin{aligned}
& \Pi_{Y_{1}} F\left(x^{*}+t h+t^{2} \bar{h}\right)=o\left(t^{2}\right), \\
& \Pi_{Y_{2}} F\left(x^{*}+t h+t^{2} \bar{h}\right)=o\left(t^{3}\right), \\
& P_{Y_{2}^{\perp}} F\left(x^{*}+t h+t^{2} \bar{h}\right)=o\left(t^{4}\right),
\end{aligned}
$$

as $t \rightarrow 0$.

Assume that the operator

$$
\begin{aligned}
\Psi_{2}\left(t h, t^{2} \bar{h}\right)= & \Pi_{Y_{1}} F^{\prime}\left(x^{*}\right)+\Pi_{Y_{2}} F^{\prime \prime}\left(x^{*}\right)[t h] \\
& +P_{Y_{2}^{\perp}}\left(F^{\prime \prime}\left(x^{*}\right)\left[t^{2} \bar{h}\right]+\frac{1}{2} F^{\prime \prime \prime}\left(x^{*}\right)[t h]^{2}\right), \quad t \neq 0,
\end{aligned}
$$

is surjective. Then there exists a curve $x^{*}+t h+t^{2} \bar{h}+x(t)$, such that $F\left(x^{*}+t h+t^{2} \bar{h}+x(t)\right)=0$ and $\|x(t)\|=o\left(t^{2}\right)$ (hence $\left.h \in T_{x^{*}} M\right)$.

Remark 22 The operator $\Psi_{2}\left(t h, t^{2} \bar{h}\right)$ will be called the generalized or modified 2-factor operator.

Proof Define a multivalued mapping

$$
\begin{aligned}
& \Phi: U(0, \varepsilon) \rightarrow 2^{Y}, \\
& \forall x \in U(0, \varepsilon), \quad \Phi(x)=\Phi(t, x)=x-\left\{\Psi_{2}\left(t h, t^{2} \bar{h}\right)\right\}^{-1} F\left(x^{*}+t h+t^{2} \bar{h}+x\right),
\end{aligned}
$$

where $t \in(-\delta, \delta) \backslash\{0\}, \delta>0$. We will show that, for any $t$ small enough, there exists an element $r(t)$ such that $\|r(t)\|=o\left(t^{2}\right)$ and $r(t) \in \Phi(r(t))$, i.e., $r(t)$ is a fixed point of the mapping $\Phi$. It follows that

$$
0 \in\left\{-\left\{\Psi_{2}\left(t h, t^{2} \bar{h}\right)\right\}^{-1} F\left(x^{*}+t h+t^{2} \bar{h}+r(t)\right)\right\}
$$

From this relation we will obtain $F\left(x^{*}+t h+t^{2} \bar{h}+r(t)\right)=0$ and $\|r(t)\|=o\left(t^{2}\right)$, hence $h \in$ $T_{x^{*}} M$.

First we will prove that

$$
\varrho(0, \Phi(0))=\|\Phi(0)\|=o\left(t^{2}\right)
$$


Let us note that

$$
\begin{aligned}
\Phi(0)= & -\left\{\Psi_{2}\left(t h, t^{2} \bar{h}\right)\right\}^{-1} F\left(x^{*}+t h+t^{2} \bar{h}\right) \\
= & -\left\{\Psi_{2}\left(t h, t^{2} \bar{h}\right)\right\}^{-1}\left(\Pi_{Y_{1}} F\left(x^{*}+t h+t^{2} \bar{h}\right)+\Pi_{Y_{2}} F\left(x^{*}+t h+t^{2} \bar{h}\right)\right. \\
& \left.+P_{Y_{2}^{\perp}} F\left(x^{*}+t h+t^{2} \bar{h}\right)\right) .
\end{aligned}
$$

From this, Lemma 12, and from the assumption we obtain

$$
\begin{aligned}
\|\Phi(0)\| \leq & c\left\|\Pi_{Y_{1}} F\left(x^{*}+t h+t^{2} \bar{h}\right)\right\|+\frac{c}{t}\left\|\Pi_{Y_{2}} F\left(x^{*}+t h+t^{2} \bar{h}\right)\right\| \\
& +\frac{c}{t^{2}}\left\|P_{Y_{2}^{\perp}} F\left(x^{*}+t h+t^{2} \bar{h}\right)\right\| \leq c o\left(t^{2}\right)+\frac{c}{t} o\left(t^{3}\right)+\frac{c}{t^{2}} o\left(t^{4}\right)=o\left(t^{2}\right) .
\end{aligned}
$$

We next show that for all $x_{1}, x_{2} \in U(0, \varepsilon(t)), \varepsilon(t)=2 o\left(t^{2}\right)$ the following estimate holds:

$$
\operatorname{dist}_{H}\left(\Phi\left(x_{1}\right), \Phi\left(x_{2}\right)\right) \leq \theta\left\|x_{1}-x_{2}\right\|, \quad 0<\theta<1 .
$$

Note that

$$
\begin{aligned}
& \Psi_{2}\left(t h, t^{2} \bar{h}\right) \Phi\left(x_{1}\right)=\Psi_{2}\left(t h, t^{2} \bar{h}\right) x_{1}-F\left(x^{*}+t h+t^{2} \bar{h}+x_{1}\right), \\
& \Psi_{2}\left(t h, t^{2} \bar{h}\right) \Phi\left(x_{2}\right)=\Psi_{2}\left(t h, t^{2} \bar{h}\right) x_{2}-F\left(x^{*}+t h+t^{2} \bar{h}+x_{2}\right) .
\end{aligned}
$$

Let $z_{1} \in \Phi\left(x_{1}\right), z_{2} \in \Phi\left(x_{2}\right)$. Then we have

$$
\begin{aligned}
& \operatorname{dist}_{H}\left(\Phi\left(x_{1}\right), \Phi\left(x_{2}\right)\right) \\
&=\inf \left\{\left\|z_{1}-z_{2}\right\|: z_{i} \in \Phi\left(x_{i}\right), i=1,2\right\} \\
&=\inf \left\{\left\|z_{1}-z_{2}\right\|: \Psi_{2}\left(t h, t^{2} \bar{h}\right)\left(z_{1}-z_{2}\right)=\Psi_{2}\left(t h, t^{2} \bar{h}\right)\left(x_{1}-x_{2}\right)\right. \\
&\left.\times\left[F\left(x^{*}+t h+t^{2} \bar{h}+x_{1}\right)-F\left(x^{*}+t h+t^{2} \bar{h}+x_{2}\right)\right]\right\} \\
&=\inf \left\{\|z\|: \Psi_{2}\left(t h, t^{2} \bar{h}\right)(z)=\Psi_{2}\left(t h, t^{2} \bar{h}\right)\left(x_{1}-x_{2}\right)\right. \\
&\left.\quad-\left[F\left(x^{*}+t h+t^{2} \bar{h}+x_{1}\right)-F\left(x^{*}+t h+t^{2} \bar{h}+x_{2}\right)\right]\right\} \\
&=\inf \left\{\|z\|: \Psi_{2}\left(t h, t^{2} \bar{h}\right)(z)=\Psi_{2}\left(t h, t^{2} \bar{h}\right)\left(x_{1}-x_{2}\right)\right. \\
&-\left[\Pi_{Y_{1}}\left(F\left(x^{*}+t h+t^{2} \bar{h}+x_{1}\right)-F\left(x^{*}+t h+t^{2} \bar{h}+x_{2}\right)\right)\right. \\
&+\Pi_{Y_{2}}\left(F\left(x^{*}+t h+t^{2} \bar{h}+x_{1}\right)-F\left(x^{*}+t h+t^{2} \bar{h}+x_{2}\right)\right) \\
&\left.\left.+P_{Y_{2}^{\perp}}\left(F\left(x^{*}+t h+t^{2} \bar{h}+x_{1}\right)-F\left(x^{*}+t h+t^{2} \bar{h}+x_{2}\right)\right)\right]\right\} \\
&=\inf \left\{\|z\|: z \in \Psi_{2}\left(t h, t^{2} \bar{h}\right)^{-1}\left\{\left[\Pi_{Y_{1}} F^{\prime}\left(x^{*}\right)\left(x_{1}-x_{2}\right)\right.\right.\right. \\
&\left.+\Pi_{Y_{2}} F^{\prime \prime}\left(x^{*}\right)[t h]\left(x_{1}-x_{2}\right)+P_{Y_{2}^{\perp}}\left(F^{\prime \prime}\left(x^{*}\right)\left[t^{2} \bar{h}\right]+\frac{1}{2} F^{\prime \prime \prime}\left(x^{*}\right)[t h]^{2}\right)\left(x_{1}-x_{2}\right)\right] \\
&+\left[\Pi_{Y_{1}}\left(F\left(x^{*}+t h+t^{2} \bar{h}+x_{1}\right)-F\left(x^{*}+t h+t^{2} \bar{h}+x_{2}\right)\right)\right. \\
&+\Pi_{Y_{2}}\left(F\left(x^{*}+t h+t^{2} \bar{h}+x_{1}\right)-F\left(x^{*}+t h+t^{2} \bar{h}+x_{2}\right)\right) \\
&\left.\left.\left.+P_{Y_{2}^{\perp}}\left(F\left(x^{*}+t h+t^{2} \bar{h}+x_{1}\right)-F\left(x^{*}+t h+t^{2} \bar{h}+x_{2}\right)\right)\right]\right\}\right\}
\end{aligned}
$$




$$
\begin{aligned}
= & \inf \left\{\| \Psi_{2}\left(t h, t^{2} \bar{h}\right)^{-1}\left\{\Pi_{Y_{1}} F^{\prime}\left(x^{*}\right)\left(x_{1}-x_{2}\right)\right.\right. \\
& -\left[\Pi_{Y_{1}}\left(F\left(x^{*}+t h+t^{2} \bar{h}+x_{1}\right)-F\left(x^{*}+t h+t^{2} \bar{h}+x_{2}\right)\right)\right] \\
& +\Pi_{Y_{2}} F^{\prime \prime}\left(x^{*}\right)[t h]\left(x_{1}-x_{2}\right) \\
& -\left[\Pi_{Y_{2}}\left(F\left(x^{*}+t h+t^{2} \bar{h}+x_{1}\right)-F\left(x^{*}+t h+t^{2} \bar{h}+x_{2}\right)\right)\right] \\
& +P_{Y_{2}^{\perp}}\left(F^{\prime \prime}\left(x^{*}\right)\left[t^{2} \bar{h}\right]+\frac{1}{2} F^{\prime \prime \prime}\left(x^{*}\right)[t h]^{2}\right)\left(x_{1}-x_{2}\right) \\
& \left.\left.-\left[P_{Y_{2}^{\perp}}\left(F\left(x^{*}+t h+t^{2} \bar{h}+x_{1}\right)-F\left(x^{*}+t h+t^{2} \bar{h}+x_{2}\right)\right)\right]\right\} \|\right\} .
\end{aligned}
$$

Further, by Lemma 12 we give the following estimate:

$$
\begin{aligned}
\operatorname{dist}_{H}( & \left.\Phi\left(x_{1}\right), \Phi\left(x_{2}\right)\right) \\
\leq & \left\|\Pi_{Y_{1}}\left(F\left(x^{*}+t h+t^{2} \bar{h}+x_{1}\right)-F\left(x^{*}+t h+t^{2} \bar{h}+x_{2}\right)\right)-\Pi_{Y_{1}} F^{\prime}\left(x^{*}\right)\left(x_{1}-x_{2}\right)\right\| \\
& +\frac{c}{t} \| \Pi_{Y_{2}}\left(F\left(x^{*}+t h+t^{2} \bar{h}+x_{1}\right)-F\left(x^{*}+t h+t^{2} \bar{h}+x_{2}\right)\right) \\
& -\Pi_{Y_{2}} F^{\prime \prime}\left(x^{*}\right)[t h]\left(x_{1}-x_{2}\right) \| \\
& +\frac{c}{t^{2}} \| P_{Y_{2}^{\perp}}\left(F\left(x^{*}+t h+t^{2} \bar{h}+x_{1}\right)-F\left(x^{*}+t h+t^{2} \bar{h}+x_{2}\right)\right) \\
& -P_{Y_{2}^{\perp}}\left(F^{\prime \prime}\left(x^{*}\right)\left[t^{2} \bar{h}\right]+\frac{1}{2} F^{\prime \prime \prime}\left(x^{*}\right)[t h]^{2}\right)\left(x_{1}-x_{2}\right) \| .
\end{aligned}
$$

Now we apply the mean value theorem and the Taylor formula. We have

$$
\begin{aligned}
\operatorname{dist}_{H} & \left(\Phi\left(x_{1}\right), \Phi\left(x_{2}\right)\right) \\
\leq & c \sup _{\bar{\theta} \in[0,1]}\left\|\Pi_{Y_{1}} F^{\prime}\left(x^{*}+t h+t^{2} \bar{h}+x_{1}+\bar{\theta}\left(x_{2}-x_{1}\right)\right)-\Pi_{Y_{1}} F^{\prime}\left(x^{*}\right)\right\|\left\|x_{1}-x_{2}\right\| \\
& +\frac{c}{t} \sup _{\bar{\theta} \in[0,1]}\left\|\Pi_{Y_{2}} F^{\prime}\left(x^{*}+t h+t^{2} \bar{h}+x_{1}+\bar{\theta}\left(x_{2}-x_{1}\right)\right)-\Pi_{Y_{2}} F^{\prime \prime}\left(x^{*}\right)[t h]\right\|\left\|x_{1}-x_{2}\right\| \\
& +\frac{c}{t^{2}} \sup _{\bar{\theta} \in[0,1]} \| P_{Y_{2}^{\perp}} F^{\prime}\left(x^{*}+t h+t^{2} \bar{h}+x_{1}+\bar{\theta}\left(x_{2}-x_{1}\right)\right) \\
& -P_{Y_{2}^{\perp}}\left(F^{\prime \prime}\left(x^{*}\right)\left[t^{2} \bar{h}\right]+\frac{1}{2} F^{\prime \prime \prime}\left(x^{*}\right)[t h]^{2}\right)\|\| x_{1}-x_{2} \| \\
= & c \sup _{\bar{\theta} \in[0,1]} \| \Pi_{Y_{1}} F^{\prime}\left(x^{*}\right)+\Pi_{Y_{1}} F^{\prime \prime}\left(x^{*}\right)\left[t h+t^{2} \bar{h}+x_{1}+\bar{\theta}\left(x_{2}-x_{1}\right)\right]+O_{Y}\left(t^{2}\right) \\
& -\Pi_{Y_{1}} F^{\prime}\left(x^{*}\right)\|\| x_{1}-x_{2} \| \\
& +\frac{c}{t} \sup _{\bar{\theta} \in[0,1]} \| \Pi_{Y_{2}} F^{\prime}\left(x^{*}\right)+\Pi_{Y_{2}} F^{\prime \prime}\left(x^{*}\right)\left[t h+t^{2} \bar{h}+x_{1}+\bar{\theta}\left(x_{2}-x_{1}\right)\right]+O_{Y}\left(t^{2}\right) \\
& -\Pi_{Y_{2}} F^{\prime \prime}\left(x^{*}\right)[t h]\|\| x_{1}-x_{2} \| \\
& +\frac{c}{t^{2}} \sup _{\bar{\theta} \in[0,1]} \| P_{Y_{2}^{\perp}} F^{\prime}\left(x^{*}\right)+P_{Y_{2}^{\perp}} F^{\prime \prime}\left(x^{*}\right)\left[t h+t^{2} \bar{h}+x_{1}+\bar{\theta}\left(x_{2}-x_{1}\right)\right] \\
& +\frac{1}{2} P_{Y_{2}^{\perp}} F^{\prime \prime \prime}\left(x^{*}\right)\left[t h+t^{2} \bar{h}+x_{1}+\bar{\theta}\left(x_{2}-x_{1}\right)\right]^{2}+O_{Y}\left(t^{3}\right)
\end{aligned}
$$




$$
\begin{aligned}
& -P_{Y_{2}^{\perp}} F^{\prime \prime}\left(x^{*}\right)\left[t^{2} \bar{h}\right]-\frac{1}{2} P_{Y_{2}^{\perp}} F^{\prime \prime \prime}\left(x^{*}\right)[t h]^{2}\|\| x_{1}-x_{2} \| \\
= & c \sup _{\bar{\theta} \in[0,1]}\left\|\Pi_{Y_{1}} F^{\prime \prime}\left(x^{*}\right)\left[t h+t^{2} \bar{h}+x_{1}+\bar{\theta}\left(x_{2}-x_{1}\right)\right]+O_{Y}\left(t^{2}\right)\right\|\left\|x_{1}-x_{2}\right\| \\
& +\frac{c}{t} \sup _{\bar{\theta} \in[0,1]}\left\|\Pi_{Y_{2}} F^{\prime \prime}\left(x^{*}\right)\left[t^{2} \bar{h}+x_{1}+\bar{\theta}\left(x_{2}-x_{1}\right)\right]+O_{Y}\left(t^{2}\right)\right\|\left\|x_{1}-x_{2}\right\| \\
& +\frac{c}{t^{2}} \sup _{\bar{\theta} \in[0,1]} \| P_{Y_{2}^{\perp}} F^{\prime \prime}\left(x^{*}\right)[t h]+P_{Y_{2}^{\perp}} F^{\prime \prime}\left(x^{*}\right)\left[t^{2} \bar{h}\right]+P_{Y_{2}^{\perp}} F^{\prime \prime}\left(x^{*}\right)\left[x_{1}+\bar{\theta}\left(x_{2}-x_{1}\right)\right] \\
& +\frac{1}{2} P_{Y_{2}^{\perp}} F^{\prime \prime \prime}\left(x^{*}\right)[t h]^{2}+\frac{1}{2} P_{Y_{2}^{\perp}} F^{\prime \prime \prime}\left(x^{*}\right)\left[t^{2} \bar{h}+x_{1}+\bar{\theta}\left(x_{2}-x_{1}\right)\right]^{2} \\
& +O_{Y}\left(t^{3}\right)-P_{Y_{2}^{\perp}} F^{\prime \prime}\left(x^{*}\right)\left[t^{2} \bar{h}\right]-\frac{1}{2} P_{Y_{2}^{\perp}} F^{\prime \prime \prime}\left(x^{*}\right)[t h]^{2}\|\| x_{1}-x_{2} \| \\
\leq & \left(\operatorname{co}(t)+\frac{c}{t} k_{1} t^{2}+\frac{c}{t^{2}} k_{2} s(t)\right)\left\|x_{1}-x_{2}\right\| \leq \theta(t)\left\|x_{1}-x_{2}\right\|,
\end{aligned}
$$

where $\theta(t) \rightarrow 0$ if $t \rightarrow 0, t \in(0, \delta)$, and $\delta>0$ is sufficiently small. Moreover, $\|s(t)\|=o\left(t^{2}\right)$. Consequently the mapping $\Phi$ is a contraction on the set $U\left(0,2 o\left(t^{2}\right)\right)$.

According to the multivalued contraction principle we will next prove that

$$
\varrho(0, \Phi(0))=\|\Phi(0)\|<(1-\theta) \varepsilon(t)
$$

where $\theta(t) \rightarrow 0$ and $\varepsilon(t)=2 o\left(t^{2}\right)$. Indeed if $t \rightarrow 0$ we can take $\theta(t)<\frac{1}{2}$, and then

$$
\|\Phi(0)\|=o\left(t^{2}\right)<(1-\theta(t)) 2 o\left(t^{2}\right)=(1-\theta(t)) \varepsilon(t) \quad(\text { since } \theta(t) \rightarrow 0),
$$

and this is the desired conclusion.

Therefore we have proved that the mapping $\Phi$ satisfies the conditions 1 and 2 of the multivalued contraction principle (Lemma 11). For $z_{0}=0$ this principle implies that there exists an element $z=r(t)$, such that $\|r(t)\| \leq \frac{2}{1-\theta}\|\Phi(0)\| \leq 4\|\Phi(0)\| \leq 8 o\left(t^{2}\right)$ or $\|r(t)\|=$ $o\left(t^{2}\right)$ and $r(t) \in \Phi(r(t))$. Then $r(t)$ is a fixed point of the mapping $\Phi$. Hence

$$
0 \in\left\{-\left\{\Psi_{2}\left(t h, t^{2} \bar{h}\right)\right\}^{-1} F\left(x^{*}+t h+t^{2} \bar{h}+r(t)\right)\right\} .
$$

Thus we get $F\left(x^{*}+t h+t^{2} \bar{h}+r(t)\right)=0$ and $\|r(t)\|=o\left(t^{2}\right)$ or $h \in T_{x^{*}} M$ and this finishes the proof.

In the next section, we apply the above theorem to a nonlinear dynamical system.

\section{Applications of generalization of Andronov-Hopf theorem to nonlinear dynamics}

In this section we consider the following nonlinear dynamical system of ordinary differential equations:

$$
\frac{d u}{d t}+f(\mu, u)=\left[\begin{array}{c}
\dot{u}_{1} \\
\dot{u}_{2} \\
\dot{u}_{3} \\
\dot{u}_{4}
\end{array}\right]+\left[\begin{array}{c}
-u_{2}+\mu u_{1}+(1+\mu)\left(u_{1}+u_{2}+u_{3}+u_{4}\right)^{3} \\
u_{1}+\mu u_{2}+(1+\mu)\left(u_{1}+u_{2}+u_{3}+u_{4}\right)^{3} \\
u_{4}+\mu u_{3}+(1+\mu)\left(u_{1}+u_{2}+u_{3}+u_{4}\right)^{3} \\
-u_{3}+\mu u_{4}+(1+\mu)\left(u_{1}+u_{2}+u_{3}+u_{4}\right)^{3}
\end{array}\right]=\left[\begin{array}{l}
0 \\
0 \\
0 \\
0
\end{array}\right],
$$


where

$$
u=\left[\begin{array}{l}
u_{1} \\
u_{2} \\
u_{3} \\
u_{4}
\end{array}\right], \quad f(\mu, u)=\left[\begin{array}{l}
f_{1} \\
f_{2} \\
f_{3} \\
f_{4}
\end{array}\right]=\left[\begin{array}{c}
-u_{2}+\mu u_{1}+(1+\mu)\left(u_{1}+u_{2}+u_{3}+u_{4}\right)^{3} \\
u_{1}+\mu u_{2}+(1+\mu)\left(u_{1}+u_{2}+u_{3}+u_{4}\right)^{3} \\
u_{4}+\mu u_{3}+(1+\mu)\left(u_{1}+u_{2}+u_{3}+u_{4}\right)^{3} \\
-u_{3}+\mu u_{4}+(1+\mu)\left(u_{1}+u_{2}+u_{3}+u_{4}\right)^{3}
\end{array}\right],
$$

and $u(0)=u(2 \pi), \mu \in \mathbb{R}$.

An equivalent representation to the above system is

$$
\left\{\begin{array}{l}
\dot{u}_{1}-u_{2}+\mu u_{1}+(1+\mu)\left(u_{1}+u_{2}+u_{3}+u_{4}\right)^{3}=0 \\
\dot{u}_{2}+u_{1}+\mu u_{2}+(1+\mu)\left(u_{1}+u_{2}+u_{3}+u_{4}\right)^{3}=0 \\
\dot{u}_{3}+u_{4}+\mu u_{3}+(1+\mu)\left(u_{1}+u_{2}+u_{3}+u_{4}\right)^{3}=0 \\
\dot{u}_{4}-u_{3}+\mu u_{4}+(1+\mu)\left(u_{1}+u_{2}+u_{3}+u_{4}\right)^{3}=0
\end{array}\right.
$$

where $\dot{u}_{l}=\frac{d u_{l}}{d t}, u_{l}(0)=u_{l}(2 \pi), l=1,2,3,4, \mu \in \mathbb{R}$ is the parameter. The problem is now to find periodic solutions of the above system with period $2 \pi$.

Note that this example was studied by Tan in [6] by an algebraic geometry method. It is a particular case of the equation

$$
\frac{d u}{d t}+T(u)+\lambda L(u)+H(\lambda, u)+K(\lambda, u)=0
$$

where $(\lambda, u) \in R \times \mathcal{C}_{2 \pi}^{1}\left(\mathbb{R}, \mathbb{R}^{4}\right)$ and

$$
\begin{aligned}
& u=\left[\begin{array}{l}
u_{1}(t) \\
u_{2}(t) \\
u_{3}(t) \\
u_{4}(t)
\end{array}\right], \quad T=\left[\begin{array}{cccc}
-1 & -1 & 0 & 0 \\
1 & -1 & 0 & 0 \\
0 & 0 & -1 & 1 \\
0 & 0 & -1 & -1
\end{array}\right], \quad L=\left[\begin{array}{llll}
1 & 0 & 0 & 0 \\
0 & 1 & 0 & 0 \\
0 & 0 & 1 & 0 \\
0 & 0 & 0 & 1
\end{array}\right], \\
& H(\lambda, u)=\lambda\left[\begin{array}{l}
\left(u_{1}+u_{2}+u_{3}+u_{4}\right)^{3} \\
\left(u_{1}+u_{2}+u_{3}+u_{4}\right)^{3} \\
\left(u_{1}+u_{2}+u_{3}+u_{4}\right)^{3} \\
\left(u_{1}+u_{2}+u_{3}+u_{4}\right)^{3}
\end{array}\right], \quad K(\lambda, u)=0 .
\end{aligned}
$$

Taking $\lambda=\bar{\mu}+\mu=1+\mu$, we turn to the equation

$$
\frac{d u}{d t}+T(u)+(1+\mu) L(u)+H(1+\mu, u)=0,
$$

or, considering the linearity of the mappings $T$ and $L$, to the equation

$$
\frac{d u}{d t}+(T+L)(u)+\mu L(u)+H(1+\mu, u)=0,
$$

i.e.,

$$
\left[\begin{array}{c}
\dot{u}_{1} \\
\dot{u}_{2} \\
\dot{u}_{3} \\
\dot{u}_{4}
\end{array}\right]+\left[\begin{array}{c}
-u_{2} \\
u_{1} \\
u_{4} \\
-u_{3}
\end{array}\right]+\left[\begin{array}{l}
\mu u_{1} \\
\mu u_{2} \\
\mu u_{3} \\
\mu u_{4}
\end{array}\right]+(1+\mu)\left[\begin{array}{l}
\left(u_{1}+u_{2}+u_{3}+u_{4}\right)^{3} \\
\left(u_{1}+u_{2}+u_{3}+u_{4}\right)^{3} \\
\left(u_{1}+u_{2}+u_{3}+u_{4}\right)^{3} \\
\left(u_{1}+u_{2}+u_{3}+u_{4}\right)^{3}
\end{array}\right]=0
$$


Note also that for the parameter $\bar{\mu}=1$ the matrix

$$
T+L=\left[\begin{array}{cccc}
0 & -1 & 0 & 0 \\
1 & 0 & 0 & 0 \\
0 & 0 & 0 & 1 \\
0 & 0 & -1 & 0
\end{array}\right]
$$

has twice eigenvalues $\pm i$. This is also an equivalent of the matrix $L_{0}=\frac{\partial f(0,0)}{\partial u}$ in Buchner $e t$ al. [5] and in the present work, because further we will also denote the matrix $T+L$ by $L_{0}$. Therefore,

$$
\operatorname{Ker}\left(L_{0}-i I\right)=\operatorname{span}\left(w_{1}, w_{2}\right)
$$

where

$$
w_{1}=\left[\begin{array}{c}
1 \\
-i \\
1 \\
i
\end{array}\right] \text { and } \quad w_{2}=\left[\begin{array}{c}
i \\
1 \\
-i \\
1
\end{array}\right]
$$

are linearly independent eigenvectors corresponding to the eigenvalue $i$ [6].

It is worth to emphasize that the authors of $[5,6]$ search periodic solutions with period near $2 \pi$. Our purpose is to find solutions of the system (24) with a fixed period which is equal to $2 \pi$.

Below we prove the following theorem.

Theorem 23 For sufficiently small $\alpha \in(-\varepsilon, \varepsilon)$ the system (23) has nontrivial solutions of the form

$$
\begin{aligned}
x(\alpha, t)= & \left(-48 \alpha^{2}+r_{1}(\alpha), 2 \alpha \cos t+r_{2}(\alpha, t),-2 \alpha \sin t+r_{2}(\alpha, t),\right. \\
& \left.-2 \alpha \sin t+r_{3}(\alpha, t), 2 \alpha \cos t+r_{4}(\alpha, t)\right),
\end{aligned}
$$

where $\left\|r_{1}(\alpha)\right\|=o\left(\alpha^{2}\right)$ and $\left\|r_{l}(\alpha, t)\right\|=o\left(\alpha^{2}\right), l=2,3,4,5$.

Proof The system (23) can be considered as

$$
\begin{aligned}
F(x)= & F(\mu, u) \\
= & F\left(\mu, u_{1}, u_{2}, u_{3}, u_{4}\right) \\
= & \left(\dot{u}_{1}-u_{2}+\mu u_{1}+(1+\mu)\left(u_{1}+u_{2}+u_{3}+u_{4}\right)^{3},\right. \\
& \dot{u}_{2}+u_{1}+\mu u_{2}+(1+\mu)\left(u_{1}+u_{2}+u_{3}+u_{4}\right)^{3}, \\
& \dot{u}_{3}+u_{4}+\mu u_{3}+(1+\mu)\left(u_{1}+u_{2}+u_{3}+u_{4}\right)^{3}, \\
& \left.\dot{u}_{4}-u_{3}+\mu u_{4}+(1+\mu)\left(u_{1}+u_{2}+u_{3}+u_{4}\right)^{3}\right)=0,
\end{aligned}
$$

where $F \in \mathcal{C}^{3}\left(\mathbb{R} \times \mathcal{C}^{1}\left(\mathbb{R}, \mathbb{R}^{4}\right), \mathcal{C}\left(\mathbb{R}, \mathbb{R}^{4}\right)\right)$ and $u_{l}(0)=u_{l}(2 \pi), l=1,2,3,4$. Note that $x^{*}=$ $(0,0)=(0,0,0,0,0)$ is a trivial solution of this system. Let us evaluate the first derivative 
of the mapping $F$ at the point $(0,0)$,

$$
F^{\prime}(0,0)=\left[\begin{array}{ccccc}
0 & \frac{d}{d \tau} & -1 & 0 & 0 \\
0 & 1 & \frac{d}{d \tau} & 0 & 0 \\
0 & 0 & 0 & \frac{d}{d \tau} & 1 \\
0 & 0 & 0 & -1 & \frac{d}{d \tau}
\end{array}\right]:=\left(0, \frac{d}{d \tau}+L_{0}\right)
$$

where

$$
L_{0}:=\frac{\partial f(0,0)}{\partial u}=\left[\begin{array}{cccc}
0 & -1 & 0 & 0 \\
1 & 0 & 0 & 0 \\
0 & 0 & 0 & 1 \\
0 & 0 & -1 & 0
\end{array}\right] \text { and }\left(\frac{d}{d \tau}+L_{0}\right) u:=\frac{d u}{d \tau}+L_{0} u .
$$

From this we can describe the kernel of the first derivative:

$$
\operatorname{Ker} F^{\prime}(0,0)=R \oplus \operatorname{Ker}\left(\frac{d}{d \tau}+L_{0}\right)
$$

The problem is now to find the space $\operatorname{Ker}\left(\frac{d}{d \tau}+L_{0}\right)$. To this end, one has to solve the following system of equations:

$$
\frac{d u}{d \tau}+L_{0} u=0
$$

with condition $u(0)=w_{k}, k=1$ or 2 (see (26)). The solution of the above system is the following:

$$
u(\tau)=e^{-L_{0} \tau} u(0)=e^{-i \tau} u(0) .
$$

Thus we obtain

$$
\operatorname{Ker}\left(\frac{d}{d \tau}+L_{0}\right)=\operatorname{span}\left(\phi_{1}, \phi_{2}, \phi_{3}, \phi_{4}\right)
$$

where $\phi_{2 k-1}(\tau)=\operatorname{Re}\left(e^{-i \tau} w_{k}\right), \phi_{2 k}(\tau)=\operatorname{Im}\left(e^{-i \tau} w_{k}\right), k=1,2$ (see [6]).

Hence for the vector $h=\left[h_{\mu}, h_{u_{1}}, h_{u_{2}}, h_{u_{3}}, h_{u_{4}}\right] \in \mathbb{R} \times \mathcal{C}^{1}\left(\mathbb{R}, \mathbb{R}^{4}\right)$, taking into account that $0 \cdot h_{\mu}=0$ and solving the following system of differential equations:

$$
F^{\prime}(0,0)[h]=\left(\frac{d h_{u_{1}}}{d \tau}-h_{u_{2}}, \frac{d h_{u_{2}}}{d \tau}+h_{u_{1}}, \frac{d h_{u_{3}}}{d \tau}+h_{u_{4}}, \frac{d h_{u_{4}}}{d \tau}-h_{u_{3}}\right)=0,
$$

subject to the conditions $h_{u_{l}}(0)=h_{u_{l}}(2 \pi), l=1,2,3$, 4, we obtain

$$
\operatorname{Ker} F^{\prime}(0,0)=\mathbb{R} \times \operatorname{span}\left(\phi_{1}, \phi_{2}, \phi_{3}, \phi_{4}\right)=\mathbb{R} \times \operatorname{Ker} F_{u}^{\prime}(0,0),
$$

where

$$
\phi_{1}=\left[\begin{array}{c}
\cos \tau \\
-\sin \tau \\
\cos \tau \\
\sin \tau
\end{array}\right], \quad \phi_{2}=\left[\begin{array}{c}
-\sin \tau \\
-\cos \tau \\
-\sin \tau \\
\cos \tau
\end{array}\right], \quad \phi_{3}=\left[\begin{array}{c}
\sin \tau \\
\cos \tau \\
-\sin \tau \\
\cos \tau
\end{array}\right], \quad \phi_{4}=\left[\begin{array}{c}
\cos \tau \\
-\sin \tau \\
-\cos \tau \\
-\sin \tau
\end{array}\right] .
$$


Define now the space $Y_{1}=\operatorname{Im}\left(\frac{d}{d \tau}+L_{0}\right)=\left(\operatorname{Ker}\left(\frac{d}{d \tau}+L_{0}\right)^{*}\right)^{\perp}$. Note that the adjoint operator has the form $\left(\frac{d}{d \tau}+L_{0}\right)^{*}=-\frac{d}{d \tau}+L_{0}^{*}=-\frac{d}{d \tau}+L_{0}^{\top}$. A basis $\left\{\psi_{1}, \psi_{2}, \psi_{3}, \psi_{4}\right\}$ of the space $\operatorname{Ker}\left(\frac{d}{d \tau}+\right.$ $\left.L_{0}\right)^{*}$ can be found with $\left\langle\psi_{l}, \phi_{j}\right\rangle=\delta_{l j}$, where $\langle g, h\rangle=\int_{0}^{2 \pi}(g(\tau), h(\tau)) d \tau$ and $(g(\tau), h(\tau))$ is the standard scalar product in $\mathbb{R}^{4}$. Then

$$
\begin{aligned}
Y_{1} & =\left\{g \in \mathcal{C}_{2 \pi}\left(\mathbb{R}, \mathbb{R}^{4}\right):\left\langle g, \psi_{l}\right\rangle=0, l=1,2,3,4\right\} \\
& =\left\{g \in \mathcal{C}_{2 \pi}\left(\mathbb{R}, \mathbb{R}^{4}\right): \int_{0}^{2 \pi}\left(g(\tau), \psi_{l}\right) d \tau=0, l=1,2,3,4\right\} .
\end{aligned}
$$

Easy computations show that $\psi_{l}=\frac{1}{4 \pi} \phi_{l}$, for $l=1,2,3,4$, and we have the following identity of subspaces:

$\operatorname{span}\left(\psi_{1}, \psi_{2}, \psi_{3}, \psi_{4}\right)=\operatorname{span}\left(\phi_{1}, \phi_{2}, \phi_{3}, \phi_{4}\right)$

Therefore, we obtain the relation

$$
\begin{aligned}
Y_{1} & =\operatorname{Im} F^{\prime}(0,0)=\left(\operatorname{Ker} F_{u}^{\prime}(0,0)^{*}\right)^{\perp}=\left(\operatorname{Ker} F_{u}^{\prime}(0,0)\right)^{\perp} \text { and } \\
Y_{1} & =\left\{g \in \mathcal{C}_{2 \pi}\left(\mathbb{R}, \mathbb{R}^{4}\right):\left\langle g, \phi_{l}\right\rangle=0, l=1,2,3,4\right\} \\
& =\left\{g \in \mathcal{C}_{2 \pi}\left(\mathbb{R}, \mathbb{R}^{4}\right): \int_{0}^{2 \pi}\left(g(\tau), \phi_{l}\right) d \tau=0, l=1,2,3,4\right\} .
\end{aligned}
$$

Now choose the vectors

$$
\begin{aligned}
& h=\left[0, \phi_{1}+\phi_{2}+\phi_{3}+\phi_{4}\right]=2[0, \cos \tau,-\sin \tau,-\sin \tau, \cos \tau] \\
& \bar{h}=[\varepsilon, 0,0,0,0] .
\end{aligned}
$$

We proceed to show that all assumptions of Theorem 21 hold, i.e.,

$$
\begin{aligned}
& \Pi_{Y_{1}} F\left(x^{*}+\alpha h+\alpha^{2} \bar{h}\right)=o\left(\alpha^{2}\right), \\
& \Pi_{Y_{2}} F\left(x^{*}+\alpha h+\alpha^{2} \bar{h}\right)=o\left(\alpha^{3}\right), \\
& P_{Y_{2}^{\perp}} F\left(x^{*}+\alpha h+\alpha^{2} \bar{h}\right)=o\left(\alpha^{4}\right),
\end{aligned}
$$

where

$$
\begin{aligned}
& Y_{1}=\operatorname{Im} F^{\prime}\left(x^{*}\right), \quad Y_{2}=\operatorname{Im} \Pi_{Y_{1}^{\perp}} F^{\prime \prime}\left(x^{*}\right)[h], \\
& Y_{2}^{\perp}=\operatorname{Im} P_{\left(Y_{1} \oplus Y_{2}\right)^{\perp}}\left(F^{\prime \prime}\left(x^{*}\right)[\bar{h}]+\frac{1}{2} F^{\prime \prime \prime}\left(x^{*}\right)[h]^{2}\right), \quad Y=Y_{1} \oplus Y_{2} \oplus Y_{2}^{\perp} .
\end{aligned}
$$

Note that

$$
\begin{aligned}
& F\left(\alpha h+\alpha^{2} \bar{h}\right) \\
& \quad=F\left(\alpha^{2} \varepsilon, 2 \alpha \cos \tau,-2 \alpha \sin \tau,-2 \alpha \sin \tau, 2 \alpha \cos \tau\right) \\
& \quad=\left(2 \alpha^{3} \varepsilon \cos \tau+A,-2 \alpha^{3} \varepsilon \sin \tau+A,-2 \alpha^{3} \varepsilon \sin \tau+A, 2 \alpha^{3} \varepsilon \cos \tau+A\right),
\end{aligned}
$$


where $A=64 \alpha^{3}\left(1+\alpha^{2} \varepsilon\right)(\cos \tau-\sin \tau)^{3}$. Therefore, $F\left(\alpha h+\alpha^{2} \bar{h}\right)=o\left(\alpha^{2}\right)$ and from this, we have $\Pi_{Y_{1}} F\left(\alpha h+\alpha^{2} \bar{h}\right)=o\left(\alpha^{2}\right)$.

Next we show that

$$
P_{Y_{1}^{\perp}} F\left(\alpha h+\alpha^{2} \bar{h}\right)=o\left(\alpha^{4}\right)
$$

for $\bar{h}=[-48,0,0,0,0]$, where $Y_{1}^{\perp}=\operatorname{Ker} F_{u}^{\prime}(0,0)$.

The projection $P_{Y_{1}^{\perp}} F\left(\alpha h+\alpha^{2} \bar{h}\right)$ has the following form:

$$
\begin{aligned}
P_{Y_{1}^{\perp}} & F\left(\alpha^{2} \varepsilon, 2 \alpha \cos \tau,-2 \alpha \sin \tau,-2 \alpha \sin \tau, 2 \alpha \cos \tau\right) \\
= & \sum_{i=1}^{4}\left\langle F\left(\alpha^{2} \varepsilon, 2 \alpha \cos \tau,-2 \alpha \sin \tau,-2 \alpha \sin \tau, 2 \alpha \cos \tau\right), \phi_{i}\right) \phi_{i} \\
= & \phi_{1} \int_{0}^{2 \pi}\left[\left(2 \alpha^{3} \varepsilon \cos \tau+A\right) \cos \tau+\left(-2 \alpha^{3} \varepsilon \sin \tau+A\right)(-\sin \tau)\right. \\
& \left.+\left(-2 \alpha^{3} \varepsilon \sin \tau+A\right) \cos \tau+\left(2 \alpha^{3} \varepsilon \cos \tau+A\right) \sin \tau\right] d \tau \\
& +\phi_{2} \int_{0}^{2 \pi}\left[\left(2 \alpha^{3} \varepsilon \cos \tau+A\right)(-\sin \tau)+\left(-2 \alpha^{3} \varepsilon \sin \tau+A\right)(-\cos \tau)\right. \\
& \left.+\left(-2 \alpha^{3} \varepsilon \sin \tau+A\right)(-\sin \tau)+\left(2 \alpha^{3} \varepsilon \cos \tau+A\right) \cos \tau\right] d \tau \\
& +\phi_{3} \int_{0}^{2 \pi}\left[\left(2 \alpha^{3} \varepsilon \cos \tau+A\right) \sin \tau+\left(-2 \alpha^{3} \varepsilon \sin \tau+A\right) \cos \tau\right. \\
& \left.+\left(-2 \alpha^{3} \varepsilon \sin \tau+A\right)(-\sin \tau)+\left(2 \alpha^{3} \varepsilon \cos \tau+A\right) \cos \tau\right] d \tau \\
& +\phi_{4} \int_{0}^{2 \pi}\left[\left(2 \alpha^{3} \varepsilon \cos \tau+A\right) \cos \tau+\left(-2 \alpha^{3} \varepsilon \sin \tau+A\right)(-\sin \tau)\right. \\
& \left.+\left(-2 \alpha^{3} \varepsilon \sin \tau+A\right)(-\cos \tau)+\left(2 \alpha^{3} \varepsilon \cos \tau+A\right)(-\sin \tau)\right] d \tau .
\end{aligned}
$$

The last expression can be represented as

$$
\begin{aligned}
P_{Y_{1}^{\perp}} & F\left(\alpha^{2} \varepsilon, 2 \alpha \cos \tau,-2 \alpha \sin \tau,-2 \alpha \sin \tau, 2 \alpha \cos \tau\right) \\
= & \phi_{1} \int_{0}^{2 \pi}\left[2 \alpha^{3} \varepsilon+64 \alpha^{3}\left(1+\alpha^{2} \varepsilon\right)\left(2 \cos ^{4} \tau+6 \cos ^{2} \tau \sin ^{2} \tau\right)\right] d \tau \\
& +\phi_{2} \int_{0}^{2 \pi}\left[2 \alpha^{3} \varepsilon+64 \alpha^{3}\left(1+\alpha^{2} \varepsilon\right)\left(2 \sin ^{4} \tau+6 \cos ^{2} \tau \sin ^{2} \tau\right)\right] d \tau \\
& +\phi_{3} \int_{0}^{2 \pi}\left[2 \alpha^{3} \varepsilon+64 \alpha^{3}\left(1+\alpha^{2} \varepsilon\right)\left(2 \cos ^{4} \tau+6 \cos ^{2} \tau \sin ^{2} \tau\right)\right] d \tau \\
& +\phi_{4} \int_{0}^{2 \pi}\left[2 \alpha^{3} \varepsilon+64 \alpha^{3}\left(1+\alpha^{2} \varepsilon\right)\left(2 \sin ^{4} \tau+6 \cos ^{2} \tau \sin ^{2} \tau\right)\right] d \tau
\end{aligned}
$$

and condition (34) holds, if

$$
\begin{aligned}
& \phi_{1} \int_{0}^{2 \pi}\left[2 \alpha^{3} \varepsilon+64 \alpha^{3}\left(2 \cos ^{4} \tau+6 \cos ^{2} \tau \sin ^{2} \tau\right)\right] d \tau \\
& \quad+\phi_{2} \int_{0}^{2 \pi}\left[2 \alpha^{3} \varepsilon+64 \alpha^{3}\left(2 \sin ^{4} \tau+6 \cos ^{2} \tau \sin ^{2} \tau\right)\right] d \tau
\end{aligned}
$$




$$
\begin{aligned}
& +\phi_{3} \int_{0}^{2 \pi}\left[2 \alpha^{3} \varepsilon+64 \alpha^{3}\left(2 \cos ^{4} \tau+6 \cos ^{2} \tau \sin ^{2} \tau\right)\right] d \tau \\
& +\phi_{4} \int_{0}^{2 \pi}\left[2 \alpha^{3} \varepsilon+64 \alpha^{3}\left(2 \sin ^{4} \tau+6 \cos ^{2} \tau \sin ^{2} \tau\right)\right] d \tau=o\left(\alpha^{4}\right) .
\end{aligned}
$$

Therefore, we have to solve the following equation:

$$
4 \pi \varepsilon+64\left(2 \frac{3 \pi}{4}+6 \frac{\pi}{4}\right)=0
$$

and we obtain $\varepsilon=-48$. From this we have $\bar{h}=[-48,0,0,0,0]$ and condition (34) is satisfied.

By $Y_{2} \subset Y_{1}^{\perp}$ (while $\left.Y_{1}^{\perp}=\operatorname{Ker} F_{u}^{\prime}(0,0)\right)$, we have

$$
\Pi_{Y_{2}} F\left(\alpha h+\alpha^{2} \bar{h}\right)=o\left(\alpha^{3}\right)
$$

and

$$
P_{Y_{2}^{\perp}} F\left(\alpha h+\alpha^{2} \bar{h}\right)=o\left(\alpha^{4}\right)
$$

In the next step we verify that the operator

$$
\begin{aligned}
\Psi_{2}(h, \bar{h})= & \Pi_{Y_{1}} F^{\prime}(0,0)+\Pi_{Y_{2}} F^{\prime \prime}(0,0)[h] \\
& +P_{Y_{2}^{\perp}}\left(F^{\prime \prime}(0,0)[\bar{h}]+\frac{1}{2} F^{\prime \prime \prime}(0,0)[h]^{2}\right)
\end{aligned}
$$

is a surjection. Of course, this operator is

$$
\begin{aligned}
\Psi_{2}(h, \bar{h})= & F^{\prime}(0,0)+\Pi_{Y_{2}} F^{\prime \prime}(0,0)[h] \\
& +P_{Y_{2}^{\perp}}\left(F^{\prime \prime}(0,0)[\bar{h}]+\frac{1}{2} F^{\prime \prime \prime}(0,0)[h]^{2}\right) .
\end{aligned}
$$

Let us note that a consequence of Lemma 15 is the following remark. If, for any $s \in$ $(0, \delta)$, where $\delta>0$ is sufficiently small, the operator $\Pi_{Y_{2}} F^{\prime \prime}(0,0)[h]+s P_{Y_{1}^{\perp}} F^{\prime \prime}(0,0)[\bar{h}]$ is a surjection, where $\Pi_{Y_{2}}: Y \rightarrow Y_{2}$ is the projection operator from the space $Y$ onto $Y_{2}$ along $Y_{1}$ and $P_{Y_{1}^{\perp}}$ is the projection operator from the space $Y$ onto $Y_{1}^{\perp}$ along $Y_{1}$, then the operator $\Pi_{Y_{2}} F^{\prime \prime}(0,0)[h]+P_{Y_{2}^{\perp}} F^{\prime \prime}(0,0)[\bar{h}]$ is a surjection too.

To begin, note that for the vector $H=\left[h_{\mu}, h_{u_{1}}, h_{u_{2}}, h_{u_{3}}, h_{u_{4}}\right]$ we obtain (see Lemma 14)

$$
F^{\prime \prime}(0,0)[H]^{2}=2 !\left(h_{\mu} h_{u_{1}}, h_{\mu} h_{u_{2}}, h_{\mu} h_{u_{3}}, h_{\mu} h_{u_{4}}\right)
$$

and, in matrix form, we get the following representation of the operator $F^{\prime \prime}(0,0)[H]$ :

$$
F^{\prime \prime}(0,0)[H]=\left[\begin{array}{ccccc}
h_{u_{1}} & h_{\mu} & 0 & 0 & 0 \\
h_{u_{2}} & 0 & h_{\mu} & 0 & 0 \\
h_{u_{3}} & 0 & 0 & h_{\mu} & 0 \\
h_{u_{4}} & 0 & 0 & 0 & h_{\mu}
\end{array}\right]
$$


It follows that

$$
F^{\prime \prime}(0,0)[h]=\left[\begin{array}{ccccc}
2 \cos \tau & 0 & 0 & 0 & 0 \\
-2 \sin \tau & 0 & 0 & 0 & 0 \\
-2 \sin \tau & 0 & 0 & 0 & 0 \\
2 \cos \tau & 0 & 0 & 0 & 0
\end{array}\right]
$$

and

$$
F^{\prime \prime}(0,0)[\bar{h}]=\left[\begin{array}{ccccc}
0 & -48 & 0 & 0 & 0 \\
0 & 0 & -48 & 0 & 0 \\
0 & 0 & 0 & -48 & 0 \\
0 & 0 & 0 & 0 & -48
\end{array}\right]
$$

We apply Lemma 10 to examine the surjectivity of operator (35) on the kernel of the first derivative at the point $(0,0)$.

Take an arbitrary vector $\left[\lambda, v_{1}, \nu_{2}, v_{3}, v_{4}\right] \in \operatorname{Ker} F^{\prime}(0,0)$. We have $\lambda \in \mathbb{R}$ and

$$
\left[\begin{array}{l}
v_{1} \\
v_{2} \\
v_{3} \\
v_{4}
\end{array}\right]=\left[\begin{array}{c}
a \cos \tau-b \sin \tau+c \sin \tau+d \cos \tau \\
-a \sin \tau-b \cos \tau+c \cos \tau-d \sin \tau \\
a \cos \tau-b \sin \tau-c \sin \tau-d \cos \tau \\
a \sin \tau+b \cos \tau+c \cos \tau-d \sin \tau
\end{array}\right]
$$

where $a, b, c, d \in \mathbb{R}$. From this, we obtain

$$
F^{\prime}(0,0)\left[\begin{array}{c}
\lambda \\
v_{1} \\
v_{2} \\
v_{3} \\
v_{4}
\end{array}\right]=0, \quad F^{\prime \prime}(0,0)[h]\left[\begin{array}{c}
\lambda \\
v_{1} \\
v_{2} \\
v_{3} \\
v_{4}
\end{array}\right]=2 \lambda\left[\begin{array}{c}
\cos \tau \\
-\sin \tau \\
-\sin \tau \\
\cos \tau
\end{array}\right] .
$$

Next we have

$$
\Pi_{Y_{2}} F^{\prime \prime}(0,0)[h]\left(\lambda, v_{1}, v_{2}, v_{3}, v_{4}\right)=4 \pi \lambda\left(\phi_{1}+\phi_{2}+\phi_{3}+\phi_{4}\right)=4 \pi \lambda\left[\begin{array}{c}
2 \cos \tau \\
-2 \sin \tau \\
-2 \sin \tau \\
2 \cos \tau
\end{array}\right]
$$

(the calculations are analogous to (34)).

Note that the element

$$
4 \pi \lambda\left[\begin{array}{c}
2 \cos \tau \\
-2 \sin \tau \\
-2 \sin \tau \\
2 \cos \tau
\end{array}\right]
$$

belongs to $\operatorname{Ker}_{u}^{\prime}(0,0)=Y_{1}^{\perp}$. 
Hence

$$
P_{Y_{1}^{\perp}} F^{\prime \prime}(0,0)[h]\left(\lambda, v_{1}, v_{2}, v_{3}, v_{4}\right)=F^{\prime \prime}(0,0)[h]\left(\lambda, v_{1}, v_{2}, v_{3}, v_{4}\right)
$$

and $\Pi_{Y_{2}} F^{\prime \prime}(0,0)[h]\left(\lambda, v_{1}, v_{2}, v_{3}, v_{4}\right)=F^{\prime \prime}(0,0)[h]\left(\lambda, v_{1}, v_{2}, v_{3}, v_{4}\right)$, because $Y_{2} \subset Y_{1}^{\perp}$.

Consider now

$$
F^{\prime \prime}(0,0)[\bar{h}]\left[\begin{array}{c}
\lambda \\
v_{1} \\
v_{2} \\
v_{3} \\
v_{4}
\end{array}\right]=\left[\begin{array}{l}
-48 v_{1} \\
-48 v_{2} \\
-48 v_{3} \\
-48 v_{4}
\end{array}\right] \text {. }
$$

Obviously

$$
F^{\prime \prime}(0,0)[\bar{h}]\left[\begin{array}{c}
\lambda \\
v_{1} \\
v_{2} \\
v_{3} \\
v_{4}
\end{array}\right] \in \operatorname{Ker} F_{u}^{\prime}(0,0)=Y_{1}^{\perp}
$$

Hence

$$
P_{Y_{1}^{\perp}} F^{\prime \prime}(0,0)[\bar{h}]\left[\begin{array}{c}
\lambda \\
v_{1} \\
v_{2} \\
v_{3} \\
v_{4}
\end{array}\right]=F^{\prime \prime}(0,0)[\bar{h}]\left[\begin{array}{c}
\lambda \\
v_{1} \\
v_{2} \\
v_{3} \\
v_{4}
\end{array}\right] .
$$

Therefore the operator

$$
\begin{aligned}
& \Pi_{Y_{2}} F^{\prime \prime}(0,0)[h]+s P_{Y_{1}^{\perp}} F^{\prime \prime}(0,0)[\bar{h}] \\
& =F^{\prime \prime}(0,0)[h]+s F^{\prime \prime}(0,0)[\bar{h}]=\left[\begin{array}{ccccc}
\cos \tau & 48 s & 0 & 0 & 0 \\
-\sin \tau & 0 & 48 s & 0 & 0 \\
-\sin \tau & 0 & 0 & 48 s & 0 \\
\cos \tau & 0 & 0 & 0 & 48 s
\end{array}\right]
\end{aligned}
$$

is a surjection onto $Y_{1}^{\perp}$ and the operator $\Pi_{Y_{2}} F^{\prime \prime}(0,0)[h]+P_{Y_{2}^{\perp}} F^{\prime \prime}(0,0)[\bar{h}]$ is also a surjection. From this and by Lemma 10 the operator (35) is a surjection. Note that the examination of the operator $P_{Y_{2}^{\perp}}\left(\frac{1}{2} F^{\prime \prime \prime}(0,0)[h]^{2}\right)$ is not necessary.

We verified all assumptions of Theorem 21. Hence there exist nontrivial solutions of system (23). We can write them in the form $x(\alpha, t)=x^{*}+\alpha h+\alpha^{2} \bar{h}+r(\alpha)$, with $\|r(\alpha)\|=$ $o\left(\alpha^{2}\right)$, i.e.,

$$
\begin{aligned}
x(\alpha, t)= & \left(-48 \alpha^{2}+r_{1}(\alpha), 2 \alpha \cos t+r_{2}(\alpha, t),-2 \alpha \sin t+r_{2}(\alpha, t),\right. \\
& \left.-2 \alpha \sin t+r_{3}(\alpha, t), 2 \alpha \cos t+r_{4}(\alpha, t)\right),
\end{aligned}
$$

where $\left\|r_{1}(\alpha)\right\|=o\left(\alpha^{2}\right)$ and $\left\|r_{l}(\alpha, t)\right\|=o\left(\alpha^{2}\right), l=2,3,4,5$, which proves Theorem 23 . 
Remark 24 For $\mu=-48 \alpha^{2}+r_{1}(\alpha)$ such that $\mu<0$ and $\left\|r_{1}(\alpha)\right\|=o\left(\alpha^{2}\right)$ the solutions can be written as follows:

$$
\begin{aligned}
u(\mu, t)= & \left(\frac{1}{2} \sqrt{-\frac{\mu}{3}} \cos t+\bar{r}_{1}(\mu, t),-\frac{1}{2} \sqrt{-\frac{\mu}{3}} \sin t+\bar{r}_{2}(\mu, t),\right. \\
& \left.-\frac{1}{2} \sqrt{-\frac{\mu}{3}} \sin t+\bar{r}_{3}(\mu, t), \frac{1}{2} \sqrt{-\frac{\mu}{3}} \cos t+\bar{r}_{4}(\mu, t)\right),
\end{aligned}
$$

where $\left\|r_{l}(\mu, t)\right\|=o(\mu), l=1,2,3,4$.

From Theorem 23 it follows that there are no solutions in the case $\mu>0$.

\section{Comparison with other results}

In the literature many publications are known where the authors consider the classical Andronov-Hopf theorem (see, for example, Marsden and McCracken [8] or Kielhöfer [7]) and describe the bifurcation of periodic solutions from the equilibrium point of a nonlinear differential equation (or nonlinear dynamical system)

$$
\dot{u}=f(\mu, u), \quad u(0)=u(\tau),
$$

where $f: \mathbb{R} \times \mathbb{R}^{n} \rightarrow \mathbb{R}^{n}$. The Lyapunov-Schmidt reduction is often applied to this case (see Golubitsky and Langford [18], and Buchner et al. [5]). The authors seek a periodic solution near the point $(0,0)$ with period $\frac{2 \pi}{1+\tau}$ for small $\tau$. Golubitsky et al. in [19] apply the so-called constrained Liapunov-Smidt procedure to study the bifurcation structure of periodic orbits near $k: l$ resonance for both reversible and Hamiltonian systems. The authors assume that $\pm k i$ and $\pm l i$ are simple eigenvalues of the matrix $L_{0}=\frac{\partial f(0,0)}{\partial u}(0<k<l$ are coprime integers) and $\pm m i$ is not an eigenvalue of $L_{0}$ where $m \neq k, l$ is any nonnegative integer.

In our paper the $p$-regularity theory (see the work of Izmailov, Tret'yakov and Marsden [1-4]) is applied to find periodic solutions with the fixed period $2 \pi$ and we do not use the classical Andronov-Hopf condition. Moreover, the problem of multivalued eigenvalues of matrix $L_{0}$ is studied. For example, the Hopf bifurcation at multiple eigenvalues was studied by Tan in his work [6] by algebraic geometry method. Our article presents some generalization of Andronov-Hopf theorem to solve a similar problem but in a different way. It is a continuation of work by Medak and Tret'yakov [20], where the authors presented a different modification of the theorem, which gives an effective method to analyze the existence of periodic solutions of nonlinear dynamical systems. The article is also a continuation of work by Grzegorczyk et al. [21]. Compared with the latter paper, where only special cases of boundary value problems were studied, the results of the present paper allow one to study the general case of a nonlinear dynamical system. It is also worth noting that the $p$-regularity theory can be applied to the study of periodic solutions in the case of $k: l$ resonance.

\section{Conclusion}

The paper is devoted to the problem of the existence of periodic solutions of a dynamical system which can be investigated by means of $p$-regularity theory. Important results of this theory are Lemmas 18 and 19. They allow us to study the existence and uniqueness of 
solutions, each of which is determined by a vector belonging to the $p$-kernel of the $p$-factor operator. The main result is Theorem 21 , on the modified $p$-factor operator, which is a certain generalization of the Andronov-Hopf theorem. We illustrate our results by finding periodic solutions of the dynamical system of ordinary differential equations with period $2 \pi$.

\section{Competing interests}

The authors declare that they have no competing interests.

\section{Authors' contributions}

All authors read and approved the final manuscript.

\section{Author details}

'Siedlce University of Natural Sciences and Humanities, 3-go Maja 54, Siedlce, 08-110, Poland. ${ }^{2}$ System Research Institute, Polish Academy of Sciences, Newelska 6, Warsaw, 01-447, Poland. ${ }^{3}$ Dorodnicyn Computer Center, Russian Academy of Sciences, Vavilova 40, Moscow, 119991, Russia.

\section{Acknowledgements}

This work was supported by the Russian Foundation for Basic Research Grant No. 14-07-00805, by the Leading Research Schools Grant No. NSH-4640.2014.1, and by the Russian Academy of Sciences Presidium Program P-8.

Received: 29 July 2014 Accepted: 25 May 2015 Published online: 10 June 2015

\section{References}

1. Izmailov, AF, Tret'yakov, AA: Factor-Analysis of Nonlinear Mappings. Nauka, Moscow (1994) (in Russian)

2. Izmailov, AF, Tret'yakov, AA: 2-Regular Solutions of Nonlinear Problems. Theory and Numerical Methods. Nauka, Moscow (1999) (in Russian)

3. Tret'yakov, AA: The implicit function theorem in degenerate problems. Russ. Math. Surv. 42, 179-180 (1987)

4. Tret'yakov, AA, Marsden, JE: Factor analysis of nonlinear mappings: $p$-regularity theory. Commun. Pure Appl. Anal. 2(4), 425-445 (2003)

5. Buchner, M, Marsden, J, Schechter, S: Applications of the blowing-up construction and algebraic geometry to bifurcation problems. J. Differ. Equ. 48, 404-433 (1983)

6. Tan, NX: Bifurcation and Hopf bifurcation at multiple eigenvalues for equations with Lipschitz mappings. Acta Math. Vietnam. 20(2), 279-311 (1995)

7. Kielhöfer, H: Hopf bifurcation at multiple eigenvalues. Arch. Ration. Mech. Anal. 69, 53-83 (1979)

8. Marsden, JE, McCracken, M: The Hopf Bifurcation and Its Applications. Appl. Math. Sciences, vol. 19. Springer, New York (1976)

9. Brezhneva, OA, Tret'yakov, AA, Chmura, A: A modified 2-factor method for solving systems of nonlinear equations. Comput. Math. Math. Phys. 41(4), 522-532 (2001)

10. Medak, B: Development of $p$-regularity apparatus and its application to describing the structure of solution sets of degenerated differential equations. Doctoral thesis, UMCS, Lublin (2013) (in Polish)

11. Bednarczuk, E, Prusińska, A, Tret'yakov, AA: High order stability conditions for degenerate optimization problems; elements of p-regularity theory. Nonlinear Anal. 74, 836-846 (2011)

12. Grzegorczyk, W, Medak, B, Tret'yakov, AA: Generalization of $p$-regularity notion and tangent cone description in the singular case. Ann. Univ. Mariae Curie-Skłodowska, Sect. A LXVI(2), 63-79 (2012)

13. Prusińska, A, Tret'yakov, AA: On the existence of solutions to nonlinear equations involving singular mappings with non-zero p-kernel. Set-Valued Anal. 19, 399-416 (2011)

14. Szczepanik, E, Prusińska, A, Tret'yakov, AA: The $p$-factor methods for nonlinear optimization. Schedae Inform. 21, 141-157 (2012)

15. Ioffe, AD, Tihomirov, VM: Theory of Extremal Problems. North-Holland, Amsterdam (1979)

16. Brezhneva, OA, Tret'yakov, AA: Implicit function theorems for nonregular mappings in Banach spaces. Exit from singularity. In: Banach Spaces and Their Applications in Analysis, pp. 285-302. de Gruyter, Berlin (2007)

17. Alexeev, VM, Tihomirov, VM, Fomin, SV: Optimal Control. Consultants Bureau, New York (1987) (in Russian (1979))

18. Golubitsky, M, Langford, WF: Classification and unfoldings of degenerate Hopf bifurcations. J. Differ. Equ. 41, 375-415 (1981)

19. Golubitsky, M, Marsden, JE: The constrained Liapunov-Schmidt procedure and periodic orbits. Fields Inst. Commun. 4, 81-127 (1995)

20. Medak, B, Tret'yakov, AA: p-Regular nonlinear dynamics. Dokl. Math. 89(1), 112-114 (2014)

21. Grzegorczyk, W, Medak, B, Tret'yakov, AA: Application of $p$-regularity theory to nonlinear boundary value problems. Bound. Value Probl. 2013, 251 (2013). http://www.boundaryvalueproblems.com/content/2013/1/251 